\title{
Gröbner bases and algebraic geometry
}

\author{
Gert-Martin Greuel and Gerhard Pfister
}

Universität Kaiserslautern, Fachbereich Mathematik

\section{Contents}

$\begin{array}{lr}\text { Preface } & 1\end{array}$

1 Introduction by simple questions 2

$\begin{array}{lll}2 & \text { Standard bases } & \mathbf{7}\end{array}$

2.1 Monomial orderings and associated rings . . . . . . . . . 7

2.2 Standard bases and normal forms . . . . . . . . . . . 10

2.3 Syzygies and free resolutions . . . . . . . . . . . 16

3 Applications $\quad \mathbf{2 0}$

3.1 The normalization ................ 20

3.2 Singularities . . . . . . . . . . . . . . 22

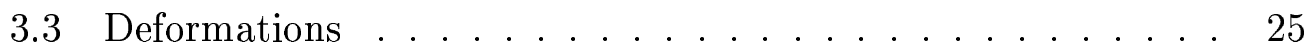

$\begin{array}{ll}\text { References } & 33\end{array}$

\section{Preface}

After the notion of Gröbner bases and an algorithm for constructing them was introduced by Buchberger [Bu1, Bu2] algebraic geometers have used Gröbner bases as the main computational tool for many years, either to prove a theorem or to disprove a conjecture or just to experiment with examples in order to obtain a feeling about the structure of an algebraic variety. Nontrivial problems coming either from logic, mathematics or applications usually lead to nontrivial Gröbner basis computations, which is the reason why several improvements have been provided by many people and have been implemented in general purpose systems like Axiom, Maple, Mathematica, Reduce, etc., and systems specialized for use in algebraic geometry and commutative algebra like CoCoA, Macaulay and Singular.

The present paper starts with an introduction to some concepts of algebraic geometry which should be understood by people with (almost) no knowledge in this field. 
In the second chapter we introduce standard bases (generalization of Gröbner bases to non-well-orderings), which are needed for applications to local algebraic geometry (singularity theory), and a method for computing syzygies and free resolutions.

The last chapter describes a new algorithm for computing the normalization of a reduced affine ring and gives an elementary introduction to singularity theory. Then we describe algorithms, using standard bases, to compute infinitesimal deformations and obstructions, which are basic for the deformation theory of isolated singularities.

It is impossible to list all papers where Gröbner bases have been used in local and global algebraic geometry, and even more impossible to give an overview about these contributions. We have, therefore, included only references to papers mentioned in this tutorial paper. The interested reader will find many more in the other contributions of this volume and in the literature cited there.

\section{Introduction by simple questions}

The basic problem of algebraic geometry can be formulated as a very simple question: "What is the structure of the set of solutions of finitely many polynomial equations in finitely many indeterminates?"

That is, we try to understand the set of points $x=\left(x_{1}, \ldots, x_{n}\right) \in K^{n}$ satisfying

$$
\begin{aligned}
& f_{1}\left(x_{1}, \ldots, x_{n}\right)=0 \\
& \vdots \\
& f_{k}\left(x_{1}, \ldots, x_{n}\right)=0
\end{aligned}
$$

where $K$ is a field and $f_{1}, \ldots, f_{k}$ are elements of the polynomial ring $K[x]=$ $K\left[x_{1}, \ldots, x_{n}\right]$. The solution set of $f_{1}, \ldots, f_{k}$ is called the algebraic set, or algebraic variety of $f_{1}, \ldots, f_{k}$ and is denoted by $V\left(f_{1}, \ldots, f_{k}\right)$.

Here are three simple examples, which will be used to illustrate some of our subsequent questions:

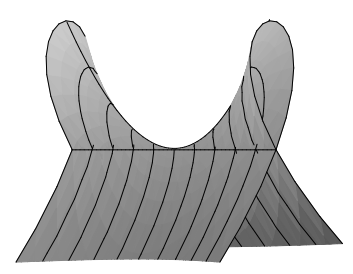

1) the hypersurface $V\left(x^{2}+y^{3}-t^{2} y^{2}\right)$

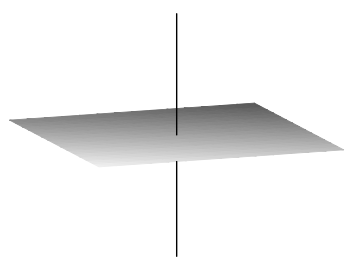

2) the variety $V(x z, y z)$

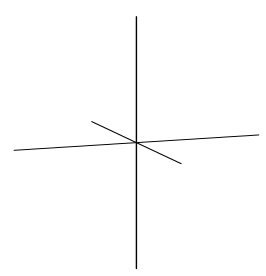

3) the variety $V(x y, x z, y z)$

The simple question, however, does not have an easy answer at all. On the contrary, the mathematical discipline algebraic geometry, which provides 
tools for possible answers, belongs with its long history to one of the highly developed branches of mathematics, which has created deep and quite sophisticated theories in geometry as well as in algebra. It has been estimated, as Kunz states in the introduction to his book on commutative algebra and algebraic geometry $[\mathrm{Ku}]$, that one can teach a course on algebraic geometry for 200 terms without repetition.

Of course, understanding is relative to the status of the theory but also to the cultural, economical and technical status of the society. Nowadays, faced by the technical revolution through computers, understanding requires, more and more, a computational approach to a problem, if possible. This is evident in algebraic geometry, as one can see, for instance, from recent textbooks (for example, [CLO], [St]). It is also evident that the majority of computational tools developed for algebraic geometry is based on Gröbner basis techniques.

Of course, any linear combination $f=\sum a_{i} f_{i}, a_{i} \in K[x]$, vanishes on $V=$ $V\left(f_{1}, \ldots, f_{k}\right)$ and $V$ is equal to the solutions of all $f \in I=\left\langle f_{1}, \ldots, f_{k}\right\rangle_{K[x]}$, the ideal generated by $f_{1}, \ldots, f_{k}$ in $K[x]$. Even the radical of $I$,

$$
\sqrt{I}=\left\{f \in K[x] \mid \exists d, f^{d} \in I\right\}
$$

has the same solution set and, by the Hilbert Nullstellensatz, there is the following tight relation between ideals of $K[x]$ and algebraic sets, provided the field $K$ is algebraically closed:

For any variety $V \subset K^{n}$ let $I(V)=\{f \in K[x] \mid f(x)=0 \forall x \in V\}$ the ideal of $V$, then

$$
V=V(J) \Rightarrow I(V)=\sqrt{J} .
$$

The converse is trivially true.

This theorem is the reason why the couple algebra and geometry married and produced so many wonderful theorems. Using this ideal-variety correspondence, we may formulate several geometric question and their algebraic counterparts.

One word about the role of the field $K$. Algebraic geometers usually draw real pictures, think about it as complex varieties and perform computations over some finite field. This attitude is justified by successful practice.

From the geometric point of view, the field $K$ is, however, extremely important. Algebraic geometry over $\mathbb{R}$, for instance, is much more complicated and by far not as complete as over $\mathbb{C}$.

The following questions and problems together with those mentioned in Buchberger's article [Bu4] belong to the very basic ones in algebraic geometry. They are also quite natural and are motivated already from the above examples. Note that for these examples, the answers are more or less obvious from the figures but, nevertheless, they require a mathematical proof which is usually given by algebra. The answers given by algebra however coincide with our geometric intuition only in the case of an algebraically closed field. 
- Is $V(I)$ irreducible or may it be decomposed into several algebraic varieties? If so, find its irreducible components. Algebraically this means to compute a primary decomposition of $I$ or of $\sqrt{I}$, the latter means to compute the associated prime ideals of $I$.

The first example is irreducible, the second has two components (one of dimension 2 and one of dimension 1), while the third example has three components (all of dimension 1).

- A natural question to ask is "How independent are the generators $f_{1}, \ldots$, $f_{k}$ of I?" that is, we ask for all relations

$$
\left(r_{1}, \ldots, r_{k}\right) \in K[x]^{r} \text {, such that } \sum r_{i} f_{i}=0 \text {. }
$$

These relations form a submodule of $K[x]^{r}$, which is called the syzygy module of $I$ and is denoted by syz $(I)$. It is the kernel of the $K[x]$-linear map

$$
K[x]^{k} \longrightarrow K[x],\left(r_{1}, \ldots, r_{k}\right) \mapsto \sum r_{i} f_{i}
$$

- More generally, we may ask for generators of the kernel of a $K[x]-$ linear map $K[x]^{r} \rightarrow K[x]^{s}$, or, in other words, for solutions of a system of linear equations over $K[x]$.

A direct geometric interpretation of syzygies is not so clear, but there are instances where properties of syzygies have important geometric consequences (cf. [Sch2]).

In example 1 we have $\operatorname{syz}(I)=0$, in example $2, \operatorname{syz}(I)=\langle(-y, x)\rangle \subset$ $K[x]^{2}$ and in example $3, \operatorname{syz}(I)=\langle(-z, y-0),(-z, 0, x)\rangle \subset K[x]^{3}$.

- A more geometric question is the following. Let $V(I)=V\left(I_{1}\right) \cup V\left(I_{2}\right)$ be a union of not necessarily irreducible varieties and let us assume that $V(I)$ and $V\left(I_{1}\right)$ are known. How can we describe $V\left(I_{2}\right)$ ? Algebraically, we want to compute generators for $I_{2}$ if we know those of $I$ and $I_{1}$. This amounts to finding generators for the ideal quotient

$$
I: I_{1}=\left\{f \in K[x] \mid f I_{1} \subset I\right\} .
$$

Geometrically, $V\left(I: I_{1}\right)$ is the smallest variety containing $V(I) \backslash V\left(I_{1}\right)$, which is the (Zariski) closure of $V(I) \backslash V\left(I_{1}\right)$.

In example 2 we have $\langle x z, y z\rangle:\langle x, y\rangle=z$ and in example $3\langle x y, x z, y z\rangle$ : $\langle x, y\rangle=\langle z, x y\rangle$, which gives, in both cases, equations for the complement of the $z$-axis $x=y=0$.

- Geometrically important is the projection of a variety $V(I) \subset K^{n}$ into a linear subspace $K^{n-r}$. Given generators $f_{1}, \ldots, f_{k}$ of $I$, we want to find 
generators for the (closure of the) image of $V(I)$ in $K^{n-r}=\left\{x \mid x_{1}=\right.$ $\left.\cdots=x_{r}=0\right\}$. The image is defined by the ideal $I \cap K\left[x_{r+1}, \ldots, x_{n}\right]$ and finding generators for this intersection is known as eliminating $x_{1}, \ldots, x_{r}$ from $f_{1}, \ldots, f_{k}$.

Projecting the three varieties above to the $(x, y)$ plane is, in the first two cases, surjective and in the third case it gives the two coordinate axes in the $(x, y)$ plane. This corresponds to the fact that the intersection with $K[x, y]$ of the first two ideals is 0 , while the last one is $x y$.

- Another problem is related to the Riemann removable theorem, which states that a function on a complex manifold, which is holomorphic and bounded outside a subvariety of codimension 1 , is actually holomorphic everywhere. This is well-known for open subsets of $\mathbb{C}$, but in higher dimension there exists a second removable theorem, which states that a function, which is holomorphic outside a subvariety of codimension 2 (no assumption on boundedness), is holomorphic everywhere.

For singular complex varieties this is not true in general, but those for which the two removable theorems hold are called normal. Moreover, each reduced variety has a normalization and there is a morphism with finite fibres from the normalization to the variety, which is an isomorphism outside the singular locus.

The problem is, given a variety $V(I) \subset K^{n}$, find a normal variety $V(J) \subset K^{m}$ and a polynomial map $K^{m} \rightarrow K^{n}$ inducing the normalization map $V(J) \rightarrow V(I)$.

The problem can be reduced to irreducible varieties (but need not be, as we shall see) and then the equivalent algebraic problem is to find the normalization of $K\left[x_{1}, \ldots, x_{n}\right] / I$, that is the integral closure of $K[x] / I$ in the quotient field of $K[x] / I$ and present this ring as an affine ring $K\left[x_{1}, \ldots, x_{m}\right] / J$ for some $m$ and $J$.

In the above examples it can be shown that the normalization of all three varieties are smooth, the last two are the disjoint union of the (smooth) components. The corresponding rings are $K\left[x_{1}, x_{2}\right], K\left[x_{1}, x_{2}\right] \oplus K\left[x_{3}\right]$, $K\left[x_{1}\right] \oplus K\left[x_{2}\right] \oplus K\left[x_{3}\right]$.

- The significance of singularities appears not only in the normalization problem. The study of singularities is also called local algebraic geometry and belongs to the basic tasks of algebraic geometry. Nowadays, singularity theory is a whole subject on its own.

A singularity of a variety is a point which has no neighbourhood in which the Jacobian matrix of the generators has constant rank.

In the first example the whole $x$-axis is singular, in the two other examples only the origin. 
One task is to compute generators for the ideal of the singular locus, which is itself a variety. This is just done by computing subdeterminants of the Jacobian matrix, if there are no components of different dimensions. In general, however, we need ideal quotients.

In the above examples, the singular locus is given by $\langle x, y\rangle,\langle x, y, z\rangle$ and $\langle x, y, z\rangle$, respectively.

- Studying a variety $V(I), I=\left(f_{1}, \ldots, f_{k}\right)$, locally at a singular point, say the origin of $K^{n}$, means studying the ideal $I K[x]_{(x)}$ generated by $I$ in the local ring

$$
K[x]_{(x)}=\left\{\frac{f}{g} \mid f, g \in K[x], g \notin\left\langle x_{1}, \ldots, x_{n}\right\rangle\right\} .
$$

In this local ring the polynomials $g$ with $g(0) \neq 0$ are units and $K[x]$ is a subring of $K[x]_{(x)}$.

Now all the problems we considered above can be formulated for ideals in $K[x]_{(x)}$ and modules over $K[x]_{(x)}$ instead of $K[x]$.

The geometric problems should be interpreted as concerning properties of the variety in a neighbourhood of the given point.

It should not be a surprise to say that all the above problems have algorithmic and computational solutions, which use, at some place, Gröbner basis methods. Moreover, algorithms for most of these have been implemented quite efficiently, in several computer algebra systems, such as CoCoA [CNR], Macaulay [GS] and SINGULAR [GPS]. The most complicated problem by far is the primary decomposition, the latest achievement is the normalization, both being implemented in SINGULAR.

At first glance, it seems that computation in the localization $K[x]_{(x)}$ requires computation with rational functions. It is an important fact that this is not necessary, but that basically the same algorithms which were developed for $K[x]$ can be used for $K[x]_{(x)}$. This is achieved by the choice of a special ordering on the monomials of $K[x]$ where, loosely speaking, the monomials of lower degree are considered to be bigger.

However, such orderings are no longer well-orderings and the classical Buchberger algorithm would not terminate. Mora discovered [Mo] that a different normal form algorithm, or, equivalently, a different division with remainders, leads to termination. Thus, Buchberger's algorithm with Mora's normal form is able to compute in $K[x]_{(x)}$ without denominators.

Several algorithms for $K[x]$ use elimination of (some auxiliary extra) variables. But variables to be eliminated have, necessarily, to be well-ordered. Hence, to be able to apply the full power of Gröbner basis methods also for the local ring $K[x]_{(x)}$, we need mixed orders, where the monomial ordering 
restricted to some variables is not a well-ordering, while restricted to other variables it is. In [GP] the authors described a modification of Mora's normal form, which terminates for mixed ordering and, more generally, for any monomial ordering which is compatible with the natural semigroup structure.

The corresponding modification of Buchberger's algorithm with this general normal form computes, in the case of a well-ordering (which we also call global ordering) Gröbner bases while, in the case of a local ordering (which was called tangent cone ordering by Mora), it computes so-called standard bases, which enjoy similar nice properties as Gröbner bases. We follow a suggestion by Mora and call bases computed by the general algorithm, standard bases, whilst, following the tradition of the last 33 years, reserving the name Gröbner basis for the established case of well-orderings.

\section{Standard bases}

Let $K$ be a field and $K[x]=K\left[x_{1}, \ldots, x_{n}\right]$ be the polynomial ring in $n$ variables over $K$.

\subsection{Monomial orderings and associated rings}

Definition 2.1. A monomial ordering on $K[x]$ is a total order on the set of monomials $\left\{x^{\alpha} \mid \alpha \in \mathbb{N}^{n}\right\}$ satisfying

$$
x^{\alpha}>x^{\beta} \Rightarrow x^{\alpha+\gamma}>x^{\beta+\gamma} \text { for all } \alpha, \beta, \gamma \in \mathbb{N}^{n} .
$$

We call a monomial ordering $>$ a global (respectively local, respectively mixed) ordering if $x_{i}>1$ for all $i$ (respectively $x_{i}<1$ for all $i$, respectively if there exist $i, j$ so that $x_{i}>1$ and $x_{j}<1$ ).

This notion is justified by the associated ring to be defined later. Note that $>$ is global if and only if $>$ is a well-ordering.

Definition 2.2. Any $f \in K[x] \backslash\{0\}$ can be written uniquely as

$$
f=c x^{\alpha}+f^{\prime}
$$

with $c \in K \backslash\{0\}$ and $\alpha>\alpha^{\prime}$ for any non-zero term $c^{\prime} x^{\alpha^{\prime}}$ of $f^{\prime}$. We set

$\operatorname{lm}(f)=x^{\alpha}$, the leading monomial of $f$, $\operatorname{lc}(f)=c$, the leading coefficient of $f$.

For a subset $G \subset K[x]$ we define the leading ideal of $G$ as

$$
L(G)=\langle\operatorname{lm}(g) \mid g \in G \backslash\{0\}\rangle_{K[x]},
$$

the ideal generated by $\{\operatorname{lm}(g) \mid g \in G \backslash\{0\}\}$ in $K[x]$. 
Typical global orderings are the lexicographical ordering lp $\left(x^{\alpha}>_{\text {lp }}\right.$ $x^{\beta}: \Leftrightarrow$ the first non-zero entry of $\alpha-\beta$ is positive) and the degree reverse lexicographical ordering $\mathbf{d p}\left(x^{\alpha}>_{\mathrm{dp}} x^{\beta}: \Leftrightarrow \operatorname{deg} x^{\alpha}>\operatorname{deg} x^{\beta}\right.$ or $\operatorname{deg} x^{\alpha}=$ $\operatorname{deg} x^{\beta}$ and the last non-zero entry of $\alpha-\beta$ is negative), typical local orderings are the negative lexicographical ordering ls $\left(x^{\alpha}>_{\mathrm{ls}} x^{\beta}: \Leftrightarrow\right.$ the first non-zero entry of $\alpha-\beta$ is negative) and the negative degree reverse lexicographical ordering $\mathbf{d s}\left(x^{\alpha}>_{\mathrm{ds}} x^{\beta}: \Leftrightarrow \operatorname{deg} x^{\alpha}<\operatorname{deg} x^{\beta}\right.$ or $\operatorname{deg} x^{\alpha}=$ $\operatorname{deg} x^{\beta}$ and the last non-zero entry of $\alpha-\beta$ is negative). In the abbreviations $\mathrm{lp}, \mathrm{dp}, \mathrm{ls}$, ds the $\mathrm{p}$ refers to a polynomial ring and the $s$ to a series ring (cf. Definition 2.3).

For practical purposes, as well as for certain theoretical arguments, it is important to extend the definitions of $d p$, respectively ds, to weighted degree orderings, where the variables have positive, respectively negative, weights.

Given monomial orderings $>_{1}$ on $K\left[x_{1}, \ldots, x_{n}\right]$ and $>_{2}$ on $K\left[y_{1}, \ldots, y_{m}\right]$, we define the product ordering or block ordering $>=\left(>_{1},>_{2}\right)$ on $K[x, y]$ by $x^{\alpha} y^{\beta}>x^{\gamma} y^{\delta}: \Leftrightarrow x^{\alpha}>_{1} x^{\gamma}$ or $x^{\alpha}=x^{\gamma}$ and $y^{\beta}>_{2} y^{\delta}$.

Definition 2.3. For a given monomial ordering $>$ define the multiplicatively closed set

$$
S_{>}:=\{u \in K[x] \backslash\{0\} \mid \operatorname{lm}(u)=1\}
$$

and the $K$-algebra

$$
\operatorname{Loc} K[x]:=S_{>}^{-1} K[x]=\left\{\frac{f}{u} \mid f \in K[x], u \in S_{>}\right\},
$$

the localization (ring of fractions) of $K[x]$ with respect to $S_{>}$.

We call Loc $K[x]$ also the ring associated to $K[x]$ and $>$.

Remark 2.1. $\quad 1) K[x] \subset \operatorname{Loc} K[x] \subset K[x]_{(x)}$ where $K[x]_{(x)}$ denotes the localization of $K[x]$ with respect to the maximal ideal $\left(x_{1}, \ldots, x_{n}\right)$. Loc $K[x]$ is Noetherian, it is $K[x]$-flat and $K[x]_{(x)}$ is Loc $K[x]$-flat.

2) $\operatorname{Loc} K[x]=K[x]$ if and only if $>$ is global and Loc $K[x]=K[x]_{(x)}$ if and only if $>$ is local (which justifies the names).

Mixed orderings occur as a product ordering of two orderings with one global and the other local. Many constructions with Gröbner bases in $K[x]$ use a set of auxiliary variables which have to be eliminated later. If one wants to perform such constructions in $K[x]_{(x)}$, the auxiliary variables must be bigger than 1 , hence, mixed orderings occur naturally in this context.

3) The product ordering on $K[x, y]=K\left[x_{1}, \ldots, x_{n}, y_{1}, \ldots, y_{m}\right]$ with $>_{1}$ global on $K[x]$ and $>_{2}$ arbitrary on $K[y]$ is an elimination ordering 
for $x_{1}, \ldots, x_{n}$ on $K[x, y]$, that is, for $g \in K[x, y]$ and $\operatorname{lm}(g) \in K[y]$ we have $g \in K[y]$. It is easy to see that for an arbitrary monomial ordering $>$ to be an elimination ordering for $x_{1}, \ldots, x_{r}$ it is necessary that $x_{i}>1$ for $i=1, \ldots, r$. For example, let $>_{1}$ be global on $K[x]$ and $>_{2}$ local on $K[y]$, then the product ordering $>=\left(>_{1},>_{2}\right)$ on $K[x, y]$ satisfies $S_{>}=K^{*}+(y) K[y]$, hence

$$
\operatorname{Loc} K[x, y]=\left(K[y]_{(y)}\right)[x]
$$

Note that $\mathrm{lm}$ and $\mathrm{lc}$ have natural extensions to the localization. For $f \in \operatorname{Loc} K[x]$ there exists $u \in S_{>}, \operatorname{lc}(u)=1$, such that $u f \in K[x]$ and we define

$$
\operatorname{lm}(f):=\operatorname{lm}(u f), \operatorname{lc}(f):=\operatorname{lc}(u f)
$$

Since

$$
\begin{aligned}
& \operatorname{lm}(f g)=\operatorname{lm}(f) \operatorname{lm}(g) \text { and } \\
& \operatorname{lc}(f g)=\operatorname{lc}(f) \operatorname{lc}(g)
\end{aligned}
$$

this definition is independent of the choice of $u$. Moreover, for a subset $G \subset$ Loc $K[x]$ set

$$
L(G)=\langle\operatorname{lm}(g) \mid g \in G \backslash\{0\}\rangle_{K[x]} \subset K[x]
$$

and call it the leading ideal of $G$. Note also that $u \in \operatorname{Loc} K[x] \backslash\{0\}$ is a unit in Loc $K[x]$ if and only if $\operatorname{lm}(u)=1$, that is, if $u \in S_{>}$.

For our intended applications of standard bases, but also for an elegant proof of Buchberger's standard basis criterion, we have to extend the notion of monomial orderings to the free module $K[x]^{r}=\sum_{i=1, \ldots, r} K[x] e_{i}$ where

$$
e_{i}=(0, \ldots, 1, \ldots, 0) \in K[x]^{r}
$$

denotes the $i$-th canonical basis vector of $K[x]^{r}$. We call

$$
x^{\alpha} e_{i}=\left(0, \ldots, x^{\alpha}, \ldots, 0\right) \in K[x]^{r}
$$

\section{a monomial (involving component i).}

Definition 2.4. Let $>$ be a monomial ordering on $K[x]$. A monomial ordering or a module ordering on $K[x]^{r}$ is a total ordering $>_{m}$ on the set of monomials $\left\{x^{\alpha} e_{i} \mid \alpha \in \mathbb{N}^{n}, i=1, \ldots, r\right\}$ satisfying

$$
\begin{array}{ll}
x^{\alpha} e_{i}>_{m} x^{\beta} e_{j} & \Rightarrow x^{\alpha+\gamma} e_{i}>_{m} x^{\beta+\gamma} e_{j}, \\
x^{\alpha}>x^{\beta} & \Rightarrow x^{\alpha} e_{i}>_{m} x^{\beta} e_{i},
\end{array}
$$


for all $\alpha, \beta, \gamma \in \mathbb{N}^{n}, i, j=1, \ldots, r$.

Two module orderings are of particular practical interest:

$$
x^{\alpha} e_{i}>x^{\beta} e_{j} \Leftrightarrow i>j \text { or } i=j \text { and } x^{\alpha}>x^{\beta},
$$

giving priority to the components and

$$
x^{\alpha} e_{i}>x^{\beta} e_{j} \Leftrightarrow x^{\alpha}>x^{\beta} \text { or } x^{\alpha}=x^{\beta} \text { and } i>j,
$$

which gives priority to the monomials in $K[x]$.

Note that, by the second condition, each component of $K[x]^{r}$ carries the ordering of $K[x]$. Hence, $>_{m}$ is a well-ordering on $K[x]^{r}$ if and only if $>$ is a well-ordering on $K[x]$. We call $>_{m}$ global respectively local respectively mixed, if this holds for $>$ respectively.

Now we fix a module ordering $>_{m}$ and denote it also with $>$. Since any $f \in K[x]^{r} \backslash\{0\}$ can be written uniquely as

$$
f=c x^{\alpha} e_{i}+f^{\prime}
$$

with $c \in K \backslash\{0\}$ and $x^{\alpha} e_{i}>x^{\alpha^{\prime}} e_{j}$ for any non-zero term $c^{\prime} x^{\alpha^{\prime}} e_{j}$ of $f^{\prime}$ we can define as before

$$
\begin{aligned}
\operatorname{lm}(f) & =x^{\alpha} e_{i}, \\
\operatorname{lc}(f) & =c
\end{aligned}
$$

and call it the leading monomial respectively the leading coefficient of $f$. Moreover, for $G \subset K[x]^{r}$ we call

$$
L(G)=\langle\operatorname{lm}(g) \mid g \in G \backslash\{0\}\rangle_{K[x]} \subset K[x]^{r}
$$

the leading submodule of $G$.

As from $K[x]$ to Loc $K[x]$ these definitions carry over naturally from $K[x]^{r}$ to $(\operatorname{Loc} K[x])^{r}$.

We say that $x^{\alpha} e_{i}$ is divisible by $x^{\beta} e_{j}$ if $i=j$ and $x^{\beta} \mid x^{\alpha}$. For any set of monomials $G \subset K[x]^{r}$ and any monomial $x^{\alpha} e_{i}$, we have

$$
x^{\alpha} e_{i} \notin\langle G\rangle_{K[x]} \Leftrightarrow x^{\alpha} e_{i} \text { is not divisible by any element of } G \text {. }
$$

\subsection{Standard bases and normal forms}

Let $>$ be a fixed monomial ordering on $K[x]$. In order to have a short notation, we write

$$
R:=\operatorname{Loc} K[x]=S_{>}^{-1} K[x]
$$

to denote the localization of $K[x]$ with respect to $>$. 
We define the notion of standard basis respectively Gröbner basis and give an algorithm to compute such a basis. In the case of a well-ordering this is Buchberger's [Bu1], [Bu2], [Bu3], [Bu4] celebrated algorithm, in the general case it is a variation of Mora's tangent cone algorithm [Mo], first published in [Getal], [GP], [Gra]. We like to stress that it is important to work consequently with the ring $R$ and not with $K[x]$, even if the input is polynomial.

\section{Definition 2.5.}

1) Let $I \subset R^{r}$ be a submodule. A finite set $G \subset I$ is called a standard basis of $I$ if and only if $L(G)=L(I)$, that is, for any $f \in I \backslash\{0\}$ there exists a $g \in G$ satisfying $\operatorname{lm}(g) \mid \operatorname{lm}(f)$.

2) If the ordering is a well-ordering, then a standard basis $G$ is called a Gröbner basis. In this case $R=K[x]$ and, hence, $G \subset I \subset K[x]^{r}$.

With the above notation, we follow the suggestion of [MPT], reserving the name Gröbner basis exclusively for well-orderings.

A set $G \subset R^{r}$ is called interreduced if $0 \notin G$ and if $\operatorname{lm}(g) \notin L(G \backslash\{g\})$.

Note that any standard basis can be made interreduced by deleting successively those $g$ with $\operatorname{lm}(g) \mid \operatorname{lm}(h)$ for some $h \in G \backslash\{g\}$. An interreduced standard basis is also called minimal.

For $f \in K[x]^{r}$ and $G \subset K[x]^{r}$ we say that $f$ is reduced with respect to $\mathbf{G}$ if no monomial of $f$ is contained in $L(G)$. If $>$ is not a well-ordering, we extend this to $f \in R^{r}$ and $G \subset R^{r}$ by saying that $f$ is (completely) reduced with respect to $G$ if there exist $u_{f} \in S_{>}$, and for each $g \in G, u_{g} \in S_{>}$such that $u_{f} f, u_{g} g \in K[x]^{r}$ and $u_{f} f$ is reduced with respect to $\left\{u_{g} g \mid g \in G\right\}$.

A set $G \subset R^{r}$ is called reduced if $0 \notin G$ and if each $g \in G$ is reduced with respect to $G \backslash\{g\}$ and if, moreover, $g-\operatorname{lc}(g) \operatorname{lm}(g)$ is reduced with respect to $G$. For $>$ a well-ordering this just means that for each $g \in G \subset K[x]^{r}, \operatorname{lm}(g)$ does not divide any monomial of any element of $G \backslash\{g\}$.

We shall see later that reduced Gröbner bases do always exist, but reduced standard bases, in general, do not.

Definition 2.6. Let $\mathcal{G}$ denote the set of all finite and ordered subsets $G \subset R^{r}$.

1) A map

$$
\mathrm{NF}: R^{r} \times \mathcal{G} \rightarrow R^{r},(f, G) \mapsto \mathrm{NF}(f \mid G),
$$

is called a normal form on $R^{r}$ if, for all $f$ and $G$,

(i) $\mathrm{NF}(f \mid G) \neq 0 \Rightarrow \operatorname{lm}(\mathrm{NF}(f \mid G)) \notin L(G)$,

(ii) $f-\mathrm{NF}(f \mid G) \in\langle G\rangle_{R}$. 
$\mathrm{NF}$ is called a reduced normal form if, moreover, $\mathrm{NF}(f, G)$ is reduced with respect to $G$. NF is called a weak normal form if, instead of (ii), only the condition (ii') holds:

(ii') for each $f \in R^{r}$ and each $G \in \mathcal{G}$ there exists a unit $u \in R$, so that $u f-\mathrm{NF}(f \mid G) \in\langle G\rangle_{R}$.

2) Let $G=\left\{g_{1}, \ldots, g_{s}\right\} \in \mathcal{G}$. A representation of $f \in\langle G\rangle_{R}$,

$$
f=\sum_{i=1}^{s} a_{i} g_{i}, \quad a_{i} \in R
$$

satisfying $\operatorname{lm}(f) \geq \operatorname{lm}\left(a_{i} g_{i}\right)$, whenever both sides are defined, is called a standard representation of $f$ (with respect to $G$ ).

Remark 2.2. The reason for introducing weak normal forms is twofold. On the one hand, they are usually more easy to compute and as good as normal forms for practical applications. On the other hand, and more seriously, normal forms may not exist, while weak normal forms do. For example, it is easy to see that $f=x \in R=K[x]_{(x)}$ (with ls) does not have a normal form with respect to $G=\left\{x-x^{2}\right\}$. On the other hand, since $(1-x) f=x-x^{2}$ and $1-x$ a unit in $R, f$ is a weak normal form of itself with respect to $G$.

$\mathrm{NF}(f \mid G)$ is by no means unique. For applications (weak) normal forms are most useful if $G$ is a standard basis of $\langle G\rangle_{R}$. We shall demonstrate this with a first application, which follows immediately from the definitions.

Lemma 2.1. Let $I \subset R^{r}$ be a submodule, $G \subset I$ a standard basis of $I$ and $\mathrm{NF}(-\mid G)$ a weak normal form on $R^{r}$ with respect to $G$.

1) For any $f \in R^{r}$ we have $f \in I \Leftrightarrow \operatorname{NF}(f \mid G)=0$.

2) If $J \subset R^{r}$ is a submodule with $I \subset J$, then $L(I)=L(J)$ implies $I=J$.

3) $I=\langle G\rangle_{R}$, that is, $G$ generates $I$ as $R$-module.

4) If $\mathrm{NF}(-\mid G)$ is a reduced normal form, then it is unique.

For describing Buchberger's normal form algorithm, we need the notion of an $s$-polynomial.

Definition 2.7. Let $f, g \in R^{r} \backslash\{0\}$ with $\operatorname{lm}(f)=x^{\alpha} e_{i}$ and $\operatorname{lm}(f)=x^{\beta} e_{j}$, respectively. Let

$$
\gamma:=\operatorname{lcm}(\alpha, \beta):=\left(\max \left(\alpha_{1}, \beta_{1}\right), \ldots, \max \left(\alpha_{n}, \beta_{n}\right)\right)
$$


be the least common multiple of $x^{\alpha}$ and $x^{\beta}$ and define the s-polynomial of $f$ and $g$ to be

$$
\operatorname{spoly}(f, g):= \begin{cases}x^{\gamma-\alpha} f-\frac{\operatorname{lc}(f)}{\operatorname{lc}(g)} x^{\gamma-\beta} g, & \text { if } i=j \\ 0, & \text { if } i \neq j .\end{cases}
$$

If $\operatorname{lm}(g) \mid \operatorname{lm}(f)$, say $\operatorname{lm}(g)=x^{\beta} e_{i}, \operatorname{lm}(f)=x^{\alpha} e_{j}$, then the $s$-polynomial is especially simple,

$$
\operatorname{spoly}(f, g)=f-\frac{c(f)}{c(g)} x^{\alpha-\beta} g
$$

and $\operatorname{lm}(\operatorname{spoly}(f, g))<\operatorname{lm}(g)$. For the normal form algorithm, the $s$-polynomial will only be used in this form, while for the standard basis algorithm we need it in the general form above. In order to be able to use the same expression in both algorithms, we prefer the definition of spoly above and not the more symmetric form $\operatorname{lc}(g) x^{\gamma-\alpha} f-\operatorname{lc}(f) x^{\gamma-\beta} g$. Both are, of course, equivalent, since our ground ring $K$ is a field.

Algorithm 2.1. Assume that $>$ is a well-ordering on $K[x]^{r}$.

NFBuchBERGER $(f \mid G)$

Input: $f \in K[x]^{r}, G \in \mathcal{G}$.

Output: $h \in K[x]^{r}$, a normal form of $f$ with respect to $G$.

- $h=f$

- while $\left(h \neq 0\right.$ and $\left.G_{h}=\{g \in G|\operatorname{lm}(g)| \operatorname{lm}(h)\} \neq \emptyset\right)$

choose any $g \in G_{h}$;

$h=\operatorname{spoly}(h, g)$;

- return $h$;

For termination and correctness see [Bu4]. Note that each specific choice of "any" gives a different normal form function.

It is easy to extend NFBuchberger to a reduced normal form.

The idea of many standard basis algorithms may be formalized as follows:

Algorithm 2.2. Let $>$ be any monomial ordering on $R^{r}$ and assume that a weak normal form algorithm $\mathrm{NF}$ on $R^{r}$ is given.

$\operatorname{STANDARD}(\mathrm{G}, \mathrm{NF})$

Input: $\quad G \in \mathcal{G}$

Output: $S \in \mathcal{G}$ such that $S$ is a standard basis of the submodule $I=\langle G\rangle_{R} \subset$ $R^{r}$ 
- $S=G$;

- $P=\{(f, g) \mid f, g \in S\} \subset S \times S$

- while $(P \neq \emptyset)$

$$
\begin{aligned}
& \text { choose }(f, g) \in P ; \\
& P=P \backslash\{(f, g)\} ; \\
& h=\mathrm{NF}(\operatorname{spoly}(f, g) \mid S) ; \\
& \text { If }(h \neq 0) \\
& \quad P=P \cup\{(h, f) \mid f \in S\} ; \\
& \quad S=S \cup h ;
\end{aligned}
$$

- $\operatorname{return} S$;

To see termination of STANDARD, note that if $h \neq 0$ then $\operatorname{lm}(h) \notin L(S)$ by property 1) of NF. Hence, we obtain a strictly increasing sequence of monomial submodules of $K[x]^{r}$, which becomes stationary by Dickson's lemma or by the Noetherian property of $K[x]$. That is, after finitely many steps, we always have $\operatorname{NF}(\operatorname{spoly}(f, g) \mid S))=0$ for $(f, g) \in P$ and, after some finite time, the pairset $P$ will become empty.

Correctness follows from applying Buchberger's fundamental standard basis criterion below.

Theorem 2.1. [Buchberger's criterion] Let $I \subset R^{r}$ be a submodule and $G=$ $\left\{g_{1}, \ldots, g_{s}\right\}$ be a subset of $I$. Let $N F(-\mid G)$ be a weak normal form on $R^{r}$ with respect to $G$, satisfying: for each $f \in R^{r}$ there exists a unit $u$ such that $u f-\mathrm{NF}(f \mid G)$ has a standard representation with respect to $G$.

Then the following are equivalent

1) $G$ is a standard basis of $I$,

2) $\mathrm{NF}(f \mid G)=0$ for all $f \in I$,

3) each $f \in I$ has a standard representation with respect to $G$,

4) $G$ generates $I$ and $\operatorname{NF}\left(\operatorname{spoly}\left(g_{i}, g_{j}\right) \mid G\right)=0$ for $i, j=1, \ldots, s$.

The implications 1) $\Rightarrow 2) \Rightarrow 3$ ) $\Rightarrow 4$ ) are easy.

The implication 4$) \Rightarrow 1$ ) is the important criterion which allows the checking and construction of standard bases in finitely many steps. The proof is most easily done by using syzygies and is, therefore, postponed to the next section (Theorem 2.2).

We present now a general normal form algorithm, which works for any monomial ordering. It is basically due to Mora [Mo], with a different notion of ecart, as given in [Getal], [GP]). 
Before doing this, let us first analyze Buchberger's algorithm in the case of a local ordering.

The standard example is in one variable $x$, with $x<1, f=x$ and $G=$ $\left\{g=x-x^{2}\right\}$. We obtain

$$
x-\left(\sum_{i=0}^{\infty} x^{i}\right)\left(x-x^{2}\right)=0
$$

in $K[[x]]$, which is checked to be true, since $\sum x^{i}=\frac{1}{1-x}$ in $K[[x]]$. However, this is not a normal form in our sense, since $\sum x^{i} \notin R$.

Mora's idea was to allow more elements for reduction in order to create a standard expression of the form

$$
u f=\sum_{i=1}^{s} a_{i} g_{i}+\mathrm{NF}(f \mid G),
$$

with $u$ a unit, $a_{i} \in K[x]$ and $\operatorname{NF}(f \mid G) \in K[x]^{r}$ in the case when the input data $f$ and $G=\left\{g_{1}, \ldots, g_{s}\right\}$ are polynomial. In the above example he arrives at an expression

$$
(1-x) x=x-x^{2}
$$

instead of $x=\left(\sum_{i=0}^{\infty} x^{i}\right)\left(x-x^{2}\right)$

Definition 2.8. For a monomial $x^{\alpha} e_{i} \in K[x]^{r} \operatorname{set} \operatorname{deg} x^{\alpha} e_{i}=\operatorname{deg} x^{\alpha}=\alpha_{1}+$ $\cdots+\alpha_{n}$. For $f \in K[x]^{r} \backslash\{0\}$, let $\operatorname{deg} f$ be the maximal degree of all monomials occurring in $f$. We define the ecart of $f$ as

$$
\operatorname{ecart}(f)=\operatorname{deg} f-\operatorname{deg} \operatorname{lm}(f) .
$$

For a homogeneous $f=\sum f_{i} e_{i}$ (all components $f_{i}$ are homogeneous polynomials of the same degree), we have ecart $(f)=0$.

Algorithm 2.3. Let $>$ be any monomial ordering on $K[x]^{r}, R=S_{>}^{-1} K[x]$. $\operatorname{NFMORA}(f \mid G)$

Input: $f \in K[x]^{r}, G=\left\{g_{1}, \ldots, g_{s}\right\} \subset K[x]^{r}$

Output: $h \in K[x]^{r}$ a weak normal form of $f$ with respect to $G$. Moreover, there exists a standard representation $u f-h=\sum_{i=1}^{s} a_{i} g_{i}$ with $a_{i} \in K[x], u \in S_{>}$.

- $h=f$;

- $T=G$, 
- while $\left(h \neq 0\right.$ and $\left.T_{h}=\{g \in T|\operatorname{lm}(g)| \operatorname{lm}(h)\} \neq \emptyset\right)$

choose $g \in T_{h}$ with ecart $(g)$ minimal;

if $(\operatorname{ecart}(g)>\operatorname{ecart}(h))$

$T=T \cup\{h\}$;

$h=\operatorname{spoly}(h, g)$;

- return $h$;

If the input is homogeneous, then the ecart is always 0 and NFMorA is equal to NFBUCHBERGER. If $>$ is a well-ordering, then $\operatorname{lm}(g) \mid \operatorname{lm}(h)$ implies that $\operatorname{lm}(g) \leq \operatorname{lm}(h)$, hence $T=G$ during the algorithm. Thus, NFMora is the same as NFBuchberger, but with a special selection strategy for the elements from $G$.

For termination and correctness see [GP].

It is clear that, with a little extra storage, the algorithm does also return $u \in S_{>}$.

Algorithm 2.4. Let $>$ be any monomial ordering on $K[x]^{r}, R=S_{>}^{-1} K[x]$. STANDARD BASIS $(\mathrm{G})$

Input: $\quad G=\left\{g_{1}, \ldots, g_{s}\right\} \subset K[x]^{r}$

Output: $S=\left\{h_{1}, \ldots, h_{t}\right\} \subset K[x]^{r}$ such that $S$ is a standard basis of $I=$ $\langle G\rangle_{R} \subset R^{r}$.

- $S=\operatorname{Standard}($ G,NFMora $)$;

- return $S$;

\subsection{Syzygies and free resolutions}

Let $K$ be a field and $>$ a monomial ordering on $K[x]^{r}$. Again $R$ denotes the localization of $K[x]$ with respect to $S_{>}$.

We shall give a method, using standard bases, to compute syzygies and, more generally, free resolutions of finitely generated $R$-modules. Syzygies and free resolutions are very important objects and basic ingredients for many constructions in homological algebra and algebraic geometry. On the other hand, the use of syzygies gives a very elegant way to prove Buchberger's criterion for a standard basis. Moreover, a close inspection of the syzygies of the generators of an ideal allows detection of useless pairs during a computation of a standard basis (cf. [MM], [Ei]). Our presentation follows partly that of Schreyer [Sch1], [Schl2], cf. also [Ei]. The generalization to arbitrary monomial orderings was first formulated and proved in [Getal] and [GP].

A syzygy or a relation between $k$ elements $f_{1}, \ldots, f_{k} \in R^{r}=\underset{i=1}{\oplus} R e_{i}$ is a $k$-tuple $\left(g_{1}, \ldots, g_{k}\right) \in R^{k}$ satisfying

$$
\sum_{i=1}^{k} g_{i} f_{i}=0
$$


The set of all syzygies between $f_{1}, \ldots, f_{k}$ is a submodule of $R^{k}$. Indeed, it is the kernel of the ring homomorphism

$$
\begin{gathered}
\varphi_{1}: F_{1}:=\bigoplus_{i=1}^{k} R \varepsilon_{i} \longrightarrow F_{0}:=\bigoplus_{i=1}^{r} R e_{i}, \\
\varepsilon_{i} \longmapsto f_{i},
\end{gathered}
$$

where $e_{i}$ respectively $\varepsilon_{i}$ denote the canonical bases of $R^{r}$ respectively $R^{k} \cdot \varphi_{1}$ surjects onto $I=\left\langle f_{1}, \ldots, f_{k}\right\rangle_{R}$ and

$$
\operatorname{syz} I=\operatorname{Ker} \varphi_{1}
$$

is called the module of syzygies of $I$ with respect to the generators $f_{1}, \ldots, f_{k}$.

We shall now define a monomial ordering on $F_{1}$, which behaves perfectly well with respect to standard bases. This was first introduced and used by Schreyer [Sch1].

Set

$$
\begin{aligned}
x^{\alpha} \varepsilon_{i}>x^{\beta} \varepsilon_{j} \Leftrightarrow & \operatorname{lm}\left(x^{\alpha} f_{i}\right)>\operatorname{lm}\left(x^{\beta} f_{j}\right) \text { or } \\
& \operatorname{lm}\left(x^{\alpha} f_{i}\right)=\operatorname{lm}\left(x^{\beta} f_{j}\right) \text { and } i<j .
\end{aligned}
$$

The left-hand side $>$ is the new ordering on $F_{1}$ and the right-hand side $>$ is the ordering on $F_{0}$. In order to distinguish them, we occasionally call them $>_{1}$ respectively $>_{0} .>_{0}$ and $>_{1}$ induce the same ordering on $R$. We call the ordering $>_{1}$ the Schreyer ordering. Note that it depends on $f_{1}, \ldots, f_{k}$.

Now we are going to prove Buchberger's criterion, stating that $G=\left\{f_{1}, \ldots\right.$, $\left.f_{k}\right\}$ is a standard basis of $I$, if, for all $i<j, \operatorname{NF}\left(\operatorname{spoly}\left(f_{i}, f_{j}\right) \mid G\right)=0$. The proof uses syzygies and is basically due to Schreyer [Sch1], [Schl2], although our generalization (to general monomial orderings) seems to be simpler. It gives, at the same time, a proof of Schreyer's result that the syzygies derived from a standard representation of $\operatorname{spoly}\left(f_{i}, f_{j}\right)$ form a standard basis of syz $I$ for the Schreyer ordering.

We introduce some notations. For each $i<j$ such that $f_{i}$ and $f_{j}$ have leading term in the same component, say $\operatorname{lm}\left(f_{i}\right)=x^{\alpha i} e_{\nu}, \operatorname{lm}\left(f_{j}\right)=x^{\alpha j} e_{\nu}$, define the monomial

$$
m_{j i}:=x^{\gamma-\alpha_{i}} \in K[x],
$$

where $\gamma=\operatorname{lcm}\left(\alpha_{i}, \alpha_{j}\right)$. If $c_{i}=\operatorname{lc}\left(f_{i}\right)$ and $c_{j}=\operatorname{lc}\left(f_{j}\right)$ then

$$
m_{j i} f_{i}-\frac{c_{i}}{c_{j}} m_{i j} f_{j}=\operatorname{spoly}\left(f_{i}, f_{j}\right)
$$

Assume now that for $i<j$

$$
\operatorname{NF}\left(\operatorname{spoly}\left(f_{i}, f_{j}\right) \mid G\right)=0,
$$


for some weak normal form NF on $R^{r}$.

Then we have a standard representation

$$
m_{j i} f_{i}-\frac{c_{i}}{c_{j}} m_{i j} f_{j}=\sum_{\nu=1}^{k} a_{\nu}^{(i j)} f_{\nu}, a_{\nu}^{(i j)} \in R .
$$

Define for $i<j$ such that $\operatorname{lm}\left(f_{i}\right)$ and $\operatorname{lm}\left(f_{j}\right)$ involve the same component

$$
s_{i j}=m_{j i} \varepsilon_{i}-\frac{c_{i}}{c_{j}} m_{i j} \varepsilon_{j}-\sum_{\nu} a_{\nu}^{(i j)} \varepsilon_{\nu}
$$

Then $s_{i j} \in \operatorname{syz} I$ and it is easy to see that

Lemma 2.2. $\operatorname{lm}\left(s_{i j}\right)=m_{j i} \varepsilon_{i}$.

Theorem 2.2. Let $G=\left\{g_{1}, \ldots, g_{s}\right\}$ be a set of generators of $I \subset R^{r}$ satisfying, for some weak normal form NF on $R^{r}$,

$$
N F\left(\operatorname{spoly}\left(g_{i}, g_{j}\right) \mid G\right)=0, i<j,
$$

then the following holds:

1) $G$ is a standard basis of $I$.

2) $\left\{s_{i j}\right\}$ is a standard basis of syz I with respect to the Schreyer ordering. In particular, $\left\{s_{i j}\right\}$ generates syz $I$.

Proof. We give a proof of 1) and 2) at the time time.

Take any $f \in I$ and a preimage $g \in F_{1}$ of $f$,

$$
g=\sum_{i=1}^{s} a_{i} \varepsilon_{i}, f=\varphi(g)=\sum_{i=1}^{s} a_{i} g_{i} .
$$

This is possible as $G$ generates $I$.

In case 1 ), we assume $f \neq 0$, in case 2$) f=0$.

Consider a standard representation of $g-h$,

$$
g=\sum a_{i j} s_{i j}+h, a_{i j} \in R
$$

where $h=\sum h_{j} \varepsilon_{j} \in F_{1}$ is a normal form of $g$ with respect to $\left\{s_{i j}\right\}$ for some weak normal form on $F_{1}$ (we need only know that it exists). We have, if $h \neq 0$,

$$
\operatorname{lm}(h)=\operatorname{lm}\left(h_{\nu}\right) \cdot \varepsilon_{\nu} \text { for some } \nu
$$

and $\operatorname{lm}(h) \notin L\left(\left\{s_{i j}\right\}\right)=\left\langle\left\{m_{j i} \varepsilon_{i}\right\}\right\rangle$ by Lemma 2.2. This shows

$$
m_{j \nu} \nmid \operatorname{lm}\left(h_{\nu}\right) \text { for all } j \text {. }
$$


Since $g-h \in\left\langle\left\{s_{i j}\right\}\right\rangle \subset \operatorname{syz} I$, we obtain

$$
f=\varphi(g)=\varphi(h)=\sum h_{j} g_{j}
$$

Assume that for some $j \neq \nu, \operatorname{lm}\left(h_{j} g_{j}\right)=\operatorname{lm}\left(h_{\nu} g_{\nu}\right)$. Then $\operatorname{lm}\left(h_{\nu} g_{\nu}\right)$ is divisible by $\operatorname{lm}\left(g_{\nu}\right)$ and by $\operatorname{lm}\left(g_{j}\right)$ and hence, by

$$
\operatorname{lm}\left(g_{\nu}\right) \operatorname{lm}\left(g_{j}\right) / \operatorname{gcd}\left(\operatorname{lm}\left(g_{\nu}\right), \operatorname{lm}\left(g_{j}\right)\right)=\operatorname{lm}\left(g_{\nu}\right) m_{j \nu} .
$$

This contradicts $m_{j \nu} \nmid \operatorname{lm}\left(h_{\nu}\right)$.

In case 1) we obtain $\operatorname{lm}(f)=\operatorname{lm}\left(h_{\nu} g_{\nu}\right) \in L(G)$, in case 2) it shows that $h \neq 0$ leads to a contradiction. In case 1) $G$ is a standard basis by definition and in case 2) $\left\{s_{i j}\right\}$ is a standard basis by Theorem $\left.2.1,2\right) \Rightarrow 1$ ), which was already proved.

We shall now see, as an application, that the Hilbert syzygy theorem holds for the rings $R=S_{>}^{-1} K[x]$, stating that each finitely generated $R$-module has a free resolution of length at most $n$, the number of variables.

Lemma 2.3. Let $G=\left\{g_{1}, \ldots, g_{s}\right\}$ be a standard basis of $I \subset R^{r}$, ordered in such a way that the following holds: if $i<j$ and $\operatorname{lm}\left(g_{i}\right)=x^{\alpha_{i}} e_{\nu}, \operatorname{lm}\left(g_{j}\right)=$ $x^{\alpha_{j}} e_{\nu}$ for some $\nu$, then $\alpha_{i} \geq \alpha_{j}$ lexicographically. Let $s_{i j}$ denote the syzygies defined above. Suppose that $\operatorname{lm}\left(g_{1}\right), \ldots, \operatorname{lm}\left(g_{s}\right)$ do not depend on the variables $x_{1}, \ldots, x_{k}$. Then the $\operatorname{lm}\left(s_{i j}\right)$, taken with respect to the Schreyer ordering, do not depend on $x_{1}, \ldots, x_{k+1}$.

Proof. Given $s_{i j}$, then $i<j$ and $\operatorname{lm}\left(g_{i}\right)$ and $\operatorname{lm}\left(g_{j}\right)$ involve the same component, say $e_{\nu}$. By assumption $\operatorname{lm}\left(g_{i}\right)=x^{\alpha_{i}} e_{\nu}, \operatorname{lm}\left(g_{j}\right)=x^{\alpha_{j}} e_{\nu}$ satisfy $\alpha_{i}=$ $\left(0, \ldots, \alpha_{i, k+1}, \ldots\right) \alpha_{j}=\left(0, \ldots, \alpha_{j, k+1}, \ldots\right)$ with $\alpha_{i, k+1} \geq \alpha_{j, k+1}$. Therefore, $\operatorname{lm}\left(s_{i j}\right)=m_{j i} e_{i}, m_{j i}=x^{\operatorname{lcm}\left(\alpha_{i}, \alpha_{j}\right)-\alpha_{i}}$, does not depend on $x_{k+1}$.

Applying the lemma successively to the higher syzygy modules, we obtain (cf. [GP] for a detailed proof):

Theorem 2.3. Let $>$ be any monomial ordering on $K[x]=K\left[x_{1}, \ldots, x_{n}\right]$ and $R=S_{>}^{-1} K[x]$ be the associated ring. Then any finitely generated $R-$ module $M$ has a free resolution

$$
0 \longrightarrow F_{m} \longrightarrow F_{m-1} \longrightarrow \ldots \longrightarrow F_{0} \longrightarrow M \longrightarrow 0
$$

$F_{i}$ free $R-$ modules, of length $m \leq n$. In particular, $R$ is a regular ring.

It is clear that the methods of this section provide an algorithm to compute (non-minimal) free resolutions. This algorithm has been implemented in SINGULAR. 


\section{Applications}

\subsection{The normalization}

Here we describe an algorithm which goes back to Grauert and Remmert [GR] and was proposed by T. de Jong ([J]). There are also algorithms by Gianni, Trager $([\mathrm{GT}])$ and Vasconcelos $([\mathrm{V}])$.

The algorithm is based on the following criterion for normality:

Proposition 3.1. Let $R$ be a Noetherian reduced ring and $J$ be a radical ideal containing a non-zero divisor such that the zero set of $J, V(J)$ contains the non-normal locus of $\operatorname{Spec}(R)$. Then $R$ is normal if and only if $R=$ $\operatorname{Hom}_{R}(J, J)$.

Remark 3.1. Let $J, R$ be as in the proposition and $x$ a non-zero divisor, then

1) $x J: J=x \cdot \operatorname{Hom}_{R}(J, J)$

and, consequently,

2) $R=\operatorname{Hom}_{R}(J, J)$ if and only if $x J: J \subseteq\langle x\rangle$.

$3)$ Let $u_{0}=x, u_{1}, \ldots, u_{s}$ be generators of $x J: J$. Because of the fact that $\operatorname{Hom}_{R}(J, J)$ is a ring we have $\frac{s(s+1)}{2}$ relations $\frac{u_{i}}{x} \cdot \frac{u_{j}}{x}=\sum_{k=0}^{s} \xi_{k}^{i j} \frac{u_{k}}{x}$, $s \geq i \geq j \geq 1, \xi_{k}^{i j} \in R$ in $\frac{1}{x}(x J: J)$, which, together with the linear relations, the syzygies, between $u_{0}, \ldots, u_{s}$ define the ring structure of $\operatorname{Hom}_{R}(J, J)$ :

$$
\begin{array}{ccc}
R\left[T_{1}, \ldots, T_{s}\right] & \rightarrow \operatorname{Hom}_{R}(J, J) \\
T_{i} & \rightsquigarrow & \frac{u_{i}}{x} .
\end{array}
$$

The kernel of this map is the ideal generated by the $T_{i} T_{j}-\sum_{k=0}^{s} \xi_{k}^{i j} T_{k}$, where $T_{0}=1$, and $\sum_{k=0}^{s} \eta_{k} T_{k}$ such that $\sum_{k=0}^{s} \eta_{k} u_{k}=0$.

\section{Algorithm 3.1.} NORMAL $(I)$

Input: $\quad$ a radical ideal $I \subseteq K\left[x_{1}, \ldots, x_{n}\right]$,

Output: $s$ polynomial rings $R_{1}, \ldots, R_{s}$ and $s$ prime ideals $I_{1} \subset R_{1}, \ldots, I_{s} \subset R_{s}$ and $s$ maps $\pi_{i}: R \rightarrow R_{j}$, such that the induced map $\pi: K\left[x_{1}, \ldots, x_{n}\right] / I \rightarrow$ $R_{1} / I_{1} \times \cdots \times R_{s} / I_{s}$ is the normalization of $K\left[x_{1}, \ldots, x_{n}\right] / I$

- Result $=\emptyset$; 
- compute idempotents of $K\left[x_{1}, \ldots, x_{n}\right] / I$;

Assume $K\left[x_{1}, \ldots, x_{n}\right] / I=K\left[x_{1}, \ldots, x_{n}\right] / I_{1} \times \cdots \times K\left[x_{1}, \ldots, x_{n}\right] / I_{s}$.

- For $i=1$ to $s$ do

- compute $J=$ singular locus of $I_{i}$

- choose $f \in J \backslash I_{i}$ and compute $I_{i}: f$ to check whether $f$ is a zero divisor

- if $I_{i}: f \supsetneqq I_{i}$ then

Result $=$ Result $\cup \operatorname{NORMAL}\left(\sqrt{I_{i}, f}\right) \cup \operatorname{NORMAL}\left(I_{i}: f\right)$ else

$J=\sqrt{I_{i}, f}$

$H=f J: J$

if $H=\langle f\rangle$

Result $=$ Result $\cup\left\{K\left[x_{1}, \ldots, x_{n}\right], I_{i}\right.$, id $\}$

else

assume $H=f J: J=\left\langle f, u_{1}, \ldots, u_{s}\right\rangle$

then compute an ideal $L \subseteq K\left[x_{1}, \ldots, x_{n}, T_{1}, \ldots, T_{s}\right]$ such

that

$K\left[x_{1}, \ldots, x_{n}, T_{1}, \ldots, T_{s}\right] / L \stackrel{\sim}{\rightarrow} \operatorname{Hom}(J, J), T_{i} \rightsquigarrow \frac{u_{i}}{f}$

let $\varphi: K\left[x_{1}, \ldots, x_{n}\right] \rightarrow K\left[x_{1}, \ldots, x_{n}, T_{1}, \ldots, T_{s}\right]$ be the inclusion.

$S=\operatorname{normal}(L)$

compose the maps of $S$ with $\varphi$;

Result $=$ Result $\cup S$;

- return Result

It remains to give an algorithm to compute the idempotents.

We shall explain this for the case when the input ideal $I$ is (weighted) homogeneous with strictly positive weight.

An idempotent $e$, that is, $e^{2}-e \in I$ has to be homogeneous of degree 0 , will, therefore, not occur in the first step. It can, however, occur after one normalization loop in $\operatorname{Hom}(J, J) \simeq K\left[x_{1}, \ldots, x_{n}, T_{1}, \ldots, T_{s}\right] / L$ because some of the generators may have the same degree.

Let $T \subseteq\left\{T_{1}, \ldots, T_{s}\right\}$ be the subset of variables of degree 0 .

Then $L \cap K[T]$ is zero-dimensional because $T_{j}^{2}-\sum \xi_{k}^{j j} T_{k} \in L \cap K[T]$ for all $T_{j} \in T$ (the weights are $\geq 0$ and, therefore, $\xi_{k}^{j j} \in K, T_{k} \in T$ ).

For this situation there is an easy algorithm:

\section{Algorithm 3.2.}

IDEMPOTENTS $(I)$ 
Input: $\quad I \subseteq K\left[x_{1}, \ldots, x_{n}\right]$ a (weighted) homogeneous radical ideal, $\operatorname{deg}\left(x_{i}\right)=$ 0 for $i \leq k, \operatorname{deg}\left(x_{i}\right)>0$ for $i>k, I \cap K\left[x_{1}, \ldots, x_{k}\right] 0$-dimensional.

Output: ideals $I_{1}, \ldots, I_{s}$ such that

$$
K\left[x_{1}, \ldots, x_{n}\right] / I=K\left[x_{1}, \ldots, x_{n}\right] / I_{1} \times \cdots \times K\left[x_{1}, \ldots, x_{n}\right] / I_{s}
$$

and $I \cap K\left[x_{1}, \ldots, x_{k}\right]=\cap\left(I_{v} \cap K\left[x_{1}, \ldots, x_{k}\right]\right)$ is the prime decomposition

- Result $=\emptyset$;

- compute $J=I \cap K\left[x_{1}, \ldots, x_{k}\right]$;

- compute $J=P_{1} \cap \cdots \cap P_{s}$ the (0-dimensional) prime decomposition;

- For $i=1$ to $s$ do

- choose $g_{i} \neq 0$ in $\underset{v \neq i}{\cap} P_{v}$

- Result $=$ Result $\cup\left\{I: g_{i}\right\}$;

- return Result;

\subsection{Singularities}

The basic concepts and ideas of singularity theory are best explained over the field $\mathbb{C}$ of complex numbers, although, algebraically, most invariants make sense over arbitrary fields.

Let $U \subset \mathbb{C}^{n}$ be an open subset in the usual Euclidian topology, and $f_{1}, \ldots, f_{k}$ holomorphic (complex analytic) functions on $U$, then we may consider

$$
V=V\left(f_{1}, \ldots, f_{k}\right)=\left\{x \in U \mid f_{1}(k)=\cdots=f_{k}(x)=0\right\},
$$

the complex analytic sub-variety defined by $f_{1}, \ldots, f_{k}$ in $U$.

In practice, $f_{1}, \ldots, f_{k}$ will be polynomials, but singularity theory is interested only in the behaviour of $V\left(f_{1}, \ldots, f_{k}\right)$ in an arbitrary small neighbourhood of some point $p \in V$, that is, the germ of $V$ at $p$, which is denoted by $(V, p)$. Algebraically, this means that we are not interested in the ideal generated by $f_{1}, \ldots, f_{k}$ in the polynomial ring $\mathbb{C}\left[x_{1}, \ldots, x_{n}\right]$ but in the ideal $I$ generated by $f_{1}, \ldots, f_{k}$ in the convergent power series ring $\mathbb{C}\left\{x_{1}-p_{1}, \ldots, x_{n}-p_{n}\right\}=\mathbb{C}\{x-p\}$.

For arbitrary fields $K$, where the notion of convergence does not make sense, we consider instead the formal power series ring $K[[x]]=K\left[\left[x_{1}, \ldots, x_{n}\right]\right]$ 
and ideals $I$ generated by formal power series (in practice polynomials) $f_{1}, \ldots$, $f_{k} \in K[[x]]$. In order to have a uniform notation, we write

$$
K\langle x\rangle=K\left\langle x_{1}, \ldots, x_{n}\right\rangle
$$

to denote both $K[[x]]$ and $K\{x\}=K\left\{x_{1}, \ldots, x_{n}\right\}$ if $K$ is a complete valued field (for example, $K=\mathbb{C}$ ).

The ring $\mathcal{O}_{V, p}=\mathbb{C}\{x-p\} / I$ (respectively $K\langle x\rangle / I$ ) is called the analytic local ring of the singularity $(V, p)$.

If $f_{1}, \ldots, f_{k}$ are polynomials, we may also consider the corresponding algebraic local ring $K[x]_{(x-p)} /\left\langle f_{1}, \ldots, f_{k}\right\rangle$, where $K[x]_{(x-p)}$ is the localization of $K[x]$ in the maximal ideal $\left\langle x_{1}-p_{1}, \ldots, x_{n}-p_{n}\right\rangle$. Indeed in this ring we are able to compute standard bases (cf. Section 1).

As in the affine case, we have the Hilbert Nullstellensatz (also called the Hilbert-Rückert Nullstellensatz), stating that

$$
\sqrt{I}=I(V, p):=\left\{f \in \mathbb{C}\{x-p\}|f|_{(V, p)}=0\right\}
$$

for $I \subset \mathbb{C}\{x-p\}$ and $(V, p)$ the complex analytic germ defined by $I$.

A (complex) singularity is, by definition, nothing but a complex analytic germ $(V, p)$ (together with its analytic local ring $\mathbb{C}\{x-p\} / I) .(V, p)$ is called non-singular or regular or smooth if $\mathbb{C}\left\{x_{1}-p_{1}, \ldots, x_{n}=p_{n}\right\} / I$ is isomorphic (as local ring) to a power series ring $\mathbb{C}\left\{y_{1}, \ldots, y_{d}\right\}$ (respectively $\left.K\left\langle x_{1}, \ldots, x_{n}\right\rangle / I \cong K\left\langle y_{1}, \ldots, y_{d}\right\rangle\right)$. By the implicit function theorem, this is equivalent to the fact that $I$ has a system of generators $g_{1}, \ldots, g_{n-d}$ such that the Jacobian matrix of $g_{1}, \ldots, g_{n-d}$ has rank $n-d$ in some neighbourhood of $p$. $(V, p)$ is called an isolated singularity if there is a neighbourhood $W \subset \mathbb{C}^{n}$ of $p$ such that $W \cap(V \backslash\{p\})$ is regular everywhere.

Isolated Singularities

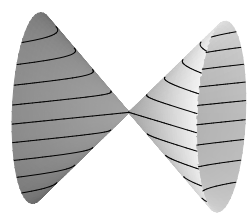

Non-isolated singularities

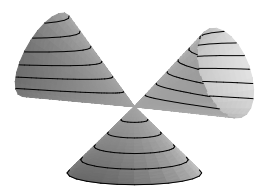

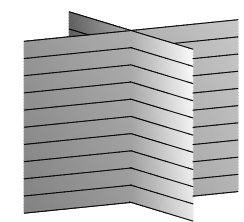

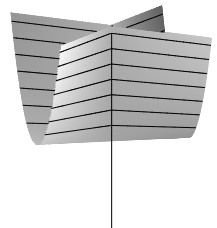

$A_{1}: x^{2}-y^{2}+z^{2}=0 D_{4}: z^{3}-z x^{2}+y^{2}=0 \quad A_{\infty}: x^{2}-y^{2}=0 D_{\infty}: y^{2}-z x^{2}=0$

The dimension of the singularity $(V, p)$ is, by definition, the Krull dimension of the analytic local ring $\mathcal{O}_{V, p}=K\langle x\rangle / I$, which is the same as the Krull dimension of the algebraic local ring $K[x]_{(x-p)} / I$ if $I=\left\langle f_{1}, \ldots, f_{k}\right\rangle$ is generated by polynomials, which follows easily from the theory of dimensions by Hilbert-Samuel series. Using this fact, we can compute $\operatorname{dim}(V, p)$ by computing a standard basis of the ideal $\left\langle f_{1}, \ldots, f_{k}\right\rangle_{\operatorname{Loc} K[x]}$ with respect to any local monomial ordering on $K[x]$. The dimension is equal to the dimension of the corresponding monomial ideal (which is a combinatorial problem). 
It is important to compute a standard basis with respect to a local ordering. For example, the leading ideal of $\langle y x-y, z x-z\rangle$, with respect to $d p$, is $\langle x y, x z\rangle$ (hence of dimension 2), but, with respect to $d s$, it is $\langle y, z\rangle$ (hence of dimension 1). Geometrically, this means that the dimension of the affine variety $V=V(y x-y, z x-z)$ is 2 but the dimension of the singularity $(V, 0)$ (that is, the dimension of $V$ at the point 0 ) is 1 :

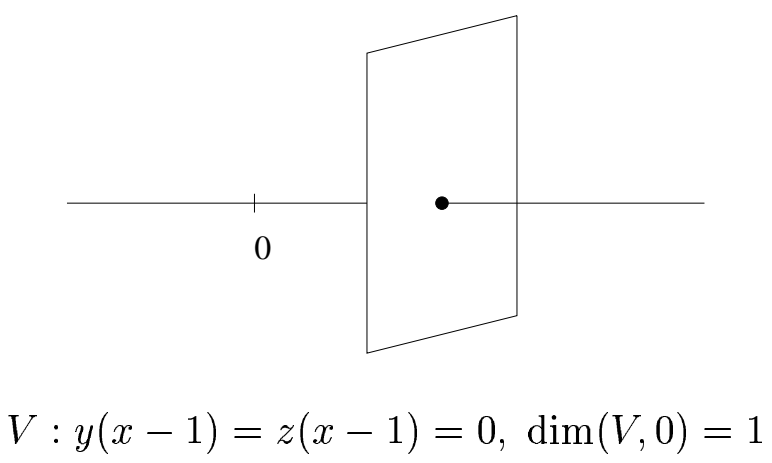

Another basic invariant is the multiplicity $\operatorname{mt}(V, p)$ of the singularity $(V, p)$. If $(V, p)$ is reduced, that is, $I=\sqrt{I}$, then $\operatorname{mt}(V, p)$ has a nice geometric interpretation: it is the number of intersection points of a sufficiently small representative $V \subset \mathbb{C}^{n}$ of $(V, p)$ with a general $(n-d)$-dimensional plane in $\mathbb{C}^{n}$ close to $p$ (but not through $p$ ). If $(V, p)$ is a hypersurface singularity, that is, $I=\langle f\rangle$ is a principal ideal in $\mathbb{C}\{x-p\}$, then $\operatorname{mt}(V, p)$ is the smallest degree in the Taylor expansion of $f$ in $p$.

Algebraically, $\operatorname{mt}(V, p)$ is the Hilbert-Samuel multiplicity of the ideal $\left\langle x_{1}-\right.$ $\left.p_{1}, \ldots, x_{n}-p_{n}\right\rangle$ in the analytic or in the algebraic local ring of $(V, p)$.

As before the Hilbert-Samuel function of the ideal defining $V$ coincides with the Hilbert-Samuel function of the leading ideal of a standard basis with respect to a local degree ordering.

One of the most important invariants of an isolated hypersurface singularity $(V, p)$ given by $I=\langle f\rangle \subset K\langle x\rangle$ is the Milnor number

$$
\mu(f):=\operatorname{dim}_{K} K\langle x\rangle /\left\langle f_{x_{1}}, \ldots, f_{x_{n}}\right\rangle
$$

$(\operatorname{char}(K)=0)$ where $f_{x_{i}}$ denotes the partial derivative of $f$ with respect to $x_{i}$.

For $K=\mathbb{C}, \mu$ is even a topological invariant and has the following topological meaning, due to Milnor [Mil]. For $f \in \mathbb{C}\left\{x_{1}, \ldots, x_{n}\right\}$ defining an isolated singularity at 0 , let

$$
V_{t}=B_{\varepsilon}(0) \cap f^{-1}(t)
$$

$0<|t|<<1$ and $B_{\varepsilon}(0)$ a small ball of radius $\varepsilon$ around 0 , then $V_{t}$ (the "Milnor fibre of $f$ ") has the homotopy type of a 1-point union of $\mu(f)(n-1)-$ dimensional spheres. In particular, $\mu(f)=\operatorname{dim}_{\mathbb{C}} H_{n}\left(V_{t}, \mathbb{C}\right)$. 
Algorithm 3.3. (Assume $\operatorname{char}(K)=0$ ). $\operatorname{MILNOR}(f)$

Input: $\quad f \in K\left[x_{1}, \ldots, x_{n}\right]$

Output: $\mu(f)$

- compute a standard basis $\left\{g_{1}, \ldots, g_{s}\right\}$ of $\left\langle f_{x_{1}}, \ldots, f_{x_{n}}\right\rangle_{\text {Loc } K[x]}$ with respect to any local monomial ordering on $K[x]$;

- the number of monomials of $K[x]$ not in $\left\langle\operatorname{lm}\left(g_{1}\right), \ldots, \operatorname{lm}\left(g_{s}\right)\right\rangle$ is equal to $\mu(f)$;

The correctness of this algorithm follows from [GP, 3.7].

Similarly, we can compute the Tjurina number $(\operatorname{char}(K) \geq 0)$

$$
\tau(f)=\operatorname{dim}_{K} K\langle x\rangle /\left\langle f, f_{x_{1}}, \ldots, f_{x_{n}}\right\rangle .
$$

This number plays an important role in the deformation theory of the singularity defined by $f$ and will be considered in the next section.

There is an interesting conjecture, due to Zariski, stating that the multiplicity of a complex hypersurface singularity is a topological invariant. This conjecture is still open. For a formulation, using the Milnor number, and for a partial positive answer (which was prompted by computer experiments using SINGULAR with local standard bases) see [GP].

\subsection{Deformations}

Let $(V, 0) \subset\left(\mathbb{C}^{n}, 0\right)$ be a singularity given by convergent power series $f_{1}, \ldots, f_{k}$, converging in a neighbourhood $U$ of $0 \in \mathbb{C}^{n}$. The idea of deformation theory is to perturb the defining functions, that is to consider functions $F_{1}(t, x), \ldots$, $F_{k}(t, x)$ with $F_{i}(0, x)=f_{i}(x)$, where $t$ are small parameters of a parameter space $S$. For $t \in S$ the functions $f_{i, t}(x)=F_{i}(t, x)$ define a complex analytic set

$$
V_{t}=V\left(f_{1, t}, \ldots, f_{k, t}\right) \subset U
$$

which, for $t$ close to 0 , may be considered to be a small deformation of $V=$ $V_{0}$. It may be hoped that $V_{t}$ is simpler than $V_{0}$ but still contains enough information about $V$. For this hope to be fulfilled, it is, however, necessary to restrict the possible perturbations of the equations to flat perturbations, which are called deformations.

The formal definition is as follows: a deformation of the singularity $(V, 0)$ over a complex analytic germ $(S, 0)$ consists of a cartesian diagram

$$
\begin{array}{cc}
(V, 0) & \stackrel{i}{\hookrightarrow}(\mathcal{U}, 0)=\left\{(t, x) \in S \times U \mid F_{1}(t, x)=\cdots=F_{k}(t, x)=0\right\} \\
\downarrow & \downarrow \\
\{0\} & \in(S, 0)
\end{array}
$$


such that $\phi$, which is the restriction of the second projection, is flat, that is, $\mathcal{O}_{\mathcal{U}, 0}$ is, via $\phi^{*}$, a flat $\mathcal{O}_{S, 0}$-module.

Grothendieck's criterion of flatness states that $\phi$ is flat if and only if any relation between the $f_{i}$, say $\sum r_{i}(x) f_{i}(x)=0$, lifts to a relation $\sum R_{i}(t, x) F_{i}(t, x)$ $=0$, where $R_{i}(x, 0)=r_{i}(x)$, between the $F_{i}$. Equivalently, for any generator $\left(r_{1}, \ldots, r_{k}\right)$ of $\operatorname{syz}\left(\left\langle f_{1}, \ldots, f_{k}\right\rangle\right)$ there exists an element $\left(R_{1}, \ldots, R_{k}\right) \in$ $\operatorname{syz}\left(\left\langle F_{1}, \ldots, F_{k}\right\rangle\right)$ satisfying $R_{i}(0, x)=r_{i}(x)$.

The notion of flatness is not easy to explain geometrically. It has, however, important geometric consequences. For example, the fibres of a flat morphism have all the same dimension. Topologists would call a flat morphism perhaps transversal. In any case, the intuitive meaning is that the fibres of a flat morphism vary in some sense continuously with the parameter.

By a theorem of Grauert [Gr] (see also Schlessinger [Schl1] for the formal case), every isolated singularity admits a semi-universal or mini-versal deformation $\phi:(\mathcal{U}, 0) \longrightarrow(S, 0)$ of $(V, 0)$, which, in some sense, contains the information upon all possible deformations.

By a power series Ansatz it is possible to compute the mini-versal deformation up to a given order. In general, the algorithm will, however, not stop. The existence of such an algorithm follows from the work of Laudal [La]. This algorithm has been implemented in SINGULAR by Martin.

We are not going to describe this algorithm here but just mention that for an isolated hypersurface singularity $f\left(x_{1}, \ldots, x_{n}\right)$ the semi-universal deformation is given by

$$
F(t, x)=f(x)+\sum_{j=1}^{\tau} t_{j} g_{j}(x),
$$

where $1=g_{1}, g_{2}, \ldots, g_{\tau}$ represent a basis of the Tjurina algebra

$$
K\langle x\rangle /\left\langle f, f_{x_{1}}, \ldots, f_{x_{n}}\right\rangle
$$

$\tau$ being the Tjurina number.

Instead we describe algorithms to compute the modules $T_{V, 0}^{1}$ respectively $T_{V, 0}^{2}$ of first order deformations of $(V, 0)$ respectively of obstructions, which are the first objects one likes to know about the semi-universal deformation.

We switch now to an algebraic setting where deformations are described on the algebra level.

Since the infinitesimal deformation theory of an affine algebra and an analytic algebra is pretty much the same (cf. $[\mathrm{Ar}]$ ), we use from now on the same notation $K\left\langle x_{1}, \ldots, x_{n}\right\rangle$ for the polynomial ring over the field $K$ as well as for power series ring over $K$.

Let $I=\left\langle f_{1}, \ldots, f_{k}\right\rangle \subset K\langle x\rangle=K\left\langle x_{1}, \ldots, x_{n}\right\rangle$ be an ideal and let $R=$ $K\langle x\rangle / I$. 
An embedded deformation of $\mathbf{R}$ over an analytic algebra $A=K\langle t\rangle / J=$ $K\left\langle t_{1}, \ldots, t_{m}\right\rangle / J$ is given by

$$
F_{i}(t, x)=f_{i}(x)+\sum_{j=1}^{m} t_{j} g_{j}^{i}(t, x) \in A\langle x\rangle
$$

satisfying that every relation (syzygy) between the $f_{i}$,

$$
\left(r_{1}, \ldots, r_{k}\right) \in K\langle x\rangle^{k}, \quad \sum_{i=1}^{k} r_{i}(x) f_{i}(x)=0,
$$

lifts to a relation between the $F_{i}$,

$$
\left(R_{1}, \ldots, R_{k}\right) \in A\langle x\rangle^{k}, \quad \sum R_{i}(t, x) F_{i}(t, x)=0,
$$

$R_{i}(0, x)=r_{i}(x)$.

By definition, $F_{1}, \ldots, F_{k}$ and $F_{1}^{\prime}, \ldots, F_{k}^{\prime}$ define the same embedded deformation, if they generate the same ideal.

Setting

$$
R_{A}=A\langle x\rangle /\left\langle F_{1}, \ldots, F_{k}\right\rangle
$$

we obtain a commutative cartesian diagram

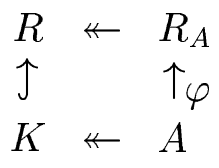

with $\varphi$ flat, which is called a deformation of $\mathbf{R}$ over $\mathbf{A}$ (and which is just the algebraic translation of the geometric definition). Two deformations $A \longrightarrow R_{A}$ and $A \longrightarrow R_{A}^{\prime}$ of $R$ over $A$ are called isomorphic if there is an $A$-isomorphism $R_{A} \cong R_{A}^{\prime}$ compatible with the given isomorphisms to the "special fibre",

$$
R_{A} /\langle t\rangle \cong R \cong R_{A}^{\prime} /\langle t\rangle,
$$

where $\langle t\rangle=\left\langle t_{1}, \ldots, t_{m}\right\rangle$. It is not difficult to see that every deformation of $R$ is isomorphic to an embedded deformation.

We like to stress the fact that the base algebras $A$ for deformations have to be local analytic $K$-algebras with $K \stackrel{\cong}{\rightrightarrows} A / \mathfrak{m}, \mathfrak{m}$ the maximal ideal of $A$, even if $R$ is affine.

\section{Infinitesimal deformations}

Let $K[\varepsilon]=K\langle\varepsilon\rangle /\left\langle\varepsilon^{2}\right\rangle$ denote the two-dimensional analytic algebra $K+K \varepsilon$, $\varepsilon^{2}=0$ (the space $\operatorname{Spec}(K[\varepsilon])$ may be considered as a "thick" point, that is, a point together with a tangent direction). An (embedded) deformation of $R$ over $K[\varepsilon]$ is called an infinitesimal (embedded) deformation. 


\section{Proposition 3.2.}

1) The $R$-module of infinitesimal embedded deformations is isomorphic to the normal module

$$
N_{R}=\operatorname{Hom}_{R}\left(I / I^{2}, R\right) .
$$

2) The $R$-module of isomorphism classes of infinitesimal deformations of $R$ is isomorphic to $T_{R}^{1}$, where $\mathbf{T}_{\mathbf{R}}^{\mathbf{1}}$ is defined by the exact sequence

$$
\Theta \otimes_{K\langle x\rangle} R \stackrel{\alpha}{\longrightarrow} N_{R} \longrightarrow T_{R}^{1} \longrightarrow 0
$$

Here, $\Theta=\operatorname{Der}_{K} K\langle x\rangle=\bigoplus_{i=1}^{n} K\langle x\rangle \frac{\partial}{\partial x_{i}}$ and the map $\alpha$ sends the derivation $\frac{\partial}{\partial x_{i}}$ to the homomorphism sending $h$ to $\frac{\partial h}{\partial x_{i}}$.

For the proof we refer to [Schl2].

\section{Remarks:}

1) Schlessinger's theorem [Schl1] states that $R$ admits a formal semi-universal deformation $B \longrightarrow R_{B}=B\langle x\rangle /\left\langle F_{1}, \ldots, F_{k}\right\rangle$ over a complete local $K-$ algebra $B$ if and only if $\operatorname{dim}_{K} T_{R}^{1}<\infty$.

This assumption is fulfilled in the affine case $R=K\left[x_{1}, \ldots, x_{n}\right] / I$ if the affine variety $V(I)$ has only isolated singularities (necessarily finitely many) or in the analytic case $R=K\left\langle x_{1}, \ldots, x_{n}\right\rangle / I$ if the singularity $(V(I), 0)$ has an isolated singularity.

2) In the complex analytic case with $R=\mathbb{C}\left\{x_{1}, \ldots, x_{n}\right\} / I$ and $\operatorname{dim}_{\mathbb{C}} T_{R}^{1}<$ $\infty, R$ admits even a convergent complex analytic semi-universal deformation with base algebra $B=\mathbb{C}\left\{t_{1}, \ldots, t_{m}\right\} / J$ and total algebra $R_{B}=\mathbb{C}\left\{t_{1}, \ldots, t_{m}, x_{1}, \ldots, x_{n}\right\} /\left\langle F_{1}, \ldots, F_{k}\right\rangle$.

The proof of the convergence is quite difficult and was given by Grauert in $1972[\mathrm{Gr}]$ and it was in this paper that he proved the "division theorem by an ideal". In our language, he introduced the notion of standard bases and proved the existence of normal forms for complex analytic convergent power series. An equivalent theorem had already been proved before in 1964 by Hironaka in his famous resolution paper ([Hi]). It is interesting to notice that the analog of Gröbner bases in power series rings was invented for proving deep theoretical results. The proofs were, however, not constructive and did not contain Buchberger's criterion.

3) It follows from Grothendieck's definition of tangent spaces that, if a semi-universal deformation $B \longrightarrow R_{B}$ of $R$ exists, then $T_{R}^{1}$ is isomorphic to the Zariski tangent space to $\operatorname{Spec} B$ at the maximal ideal of $B$. 
This shows, with $t_{1}, \ldots, t_{m}$ a $K$-basis of $T_{R}^{1 *}$, that $B \cong K\left\langle T_{R}^{1}\right\rangle / J \cong$ $K\left\langle t_{1}, \ldots, t_{m}\right\rangle / J$ for some ideal of $J$. Hence, the base algebra $B$ of the semi-universal deformation of $R$ is defined by analytic relations between the elements $t_{1}, \ldots, t_{m}$ of a $K$-basis of the dual of $T_{R}^{1}$ and these relations generate $J$.

To compute $T_{R}^{1}$, let $0 \longleftarrow R \longleftarrow K\langle x\rangle \longleftarrow K\langle x\rangle^{k} \stackrel{r}{\longleftarrow} K\langle x\rangle^{\ell}$ be a representation of $R$, then, applying $\operatorname{Hom}_{K\langle x\rangle}(-, R)$ to the sequence

$$
0 \longleftarrow R \longleftarrow K\langle x\rangle \longleftarrow K\langle x\rangle^{k} \stackrel{r}{\longleftarrow} K\langle x\rangle^{\ell}
$$

we obtain $N_{R}=\operatorname{ker}\left(R^{k} \stackrel{r^{t}}{\longrightarrow} R^{\ell}\right)$.

Now choose a resolution of $N_{R}$

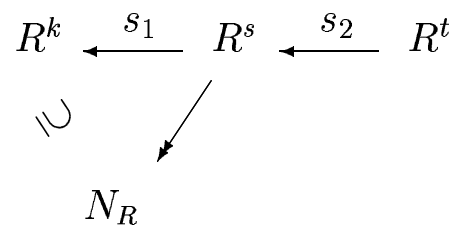

The canonical map $\pi: R^{n} \simeq \Theta_{K\langle x\rangle} \otimes R \longrightarrow N_{R}$ is induced by the map $j: R^{n} \longrightarrow R^{k}$ defined by the Jacobian matrix $\left(\frac{\partial f_{i}}{\partial x_{j}}\right)_{\substack{i<k \\ j \leq n}}$. We can lift $j$ to a map $l j: R^{n} \longrightarrow R^{s}$ such that $s_{1} \circ l j=j$ because $j$ induces the map $\pi$.

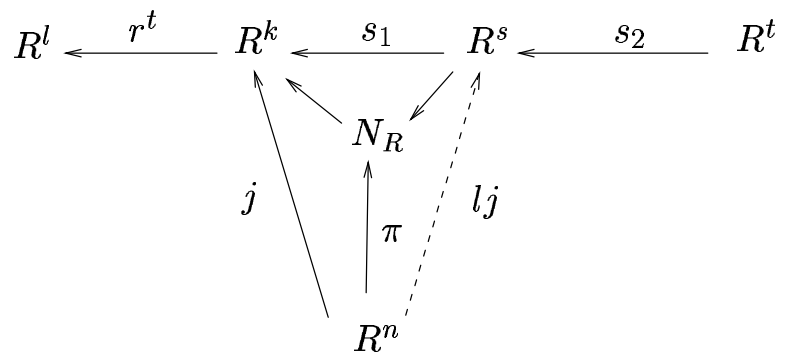

Now $T_{R}^{1}=N_{R} / \operatorname{Im}(\pi) \simeq R^{s} / \operatorname{Im}\left(s_{2}\right)+\operatorname{Im}(l j)$ gives the required representation

$$
0 \longleftarrow T_{R}^{1} \longleftarrow R^{s} \stackrel{s_{2} \oplus l j}{\longleftarrow} R^{t} \oplus R^{n}
$$

\section{Algorithm 3.4.}

$\mathrm{T} 1(I)$

Input: $\quad$ an ideal $I=\left\langle f_{1}, \ldots, f_{k}\right\rangle \subseteq K\langle x\rangle$,

Output: a matrix $M \in M_{a, b}(K\langle x\rangle)$ which defines a representation

$$
T_{K\langle x\rangle / I}^{1} \nleftarrow K\langle x\rangle^{a} \stackrel{M}{\longleftarrow} K\langle x\rangle^{b}
$$


- compute $r=\operatorname{syz}(I)$ the matrix of the syzygies of $f_{1}, \ldots, f_{k}$;

- compute the Jacobian matrix $j=\left(\frac{\partial f_{i}}{\partial x_{j}}\right)$;

- compute in $K\langle x\rangle / I$ a representation of the kernel of the transposed matrix $r^{t}$ of $r$ :

$$
(K\langle x\rangle / I)^{k} \stackrel{s_{1}}{\longleftarrow}(K\langle x\rangle / I)^{s} \stackrel{s_{2}}{\longleftarrow}(K\langle x\rangle / I)^{t} ;
$$

- lift the Jacobian matrix $j$ to a $s \times n$-matrix $l j$ such that $s_{1} \cdot l j=j$;

- concatenate $l j$ and $s_{2}$ to obtain the matrix $t 1=l j, s_{2}$;

- choose a matrix $M_{0} \in M_{s, n+t}(K\langle x\rangle)$ such that $M_{0} \bmod I=t 1$;

- choose a matrix $L \in M_{s, k \cdot s}$ (corresponding to $I K\langle x\rangle^{s}$ ), such that

$$
0 \leftarrow(K\langle x\rangle / I)^{s} \leftarrow K\langle x\rangle^{s} \stackrel{L}{\longleftarrow} K\langle x\rangle^{k \cdot s}
$$

is exact;

- concatenate $M_{0}$ and $L$ to obtain $M=M_{0}, L$;

- return $M$

\section{Obstructions}

The construction of a semi-universal deformation of $R$, in case $T_{R}^{1}$ is finite dimensional, starts with the preceding remark 3): we start with the infinitesimal deformations of first order, that is, with elements of $T_{R}^{1}$, and try to lift these to second order. This is not always possible, there are obstructions against lifting. That is, a lifting to second order is possible if and only if the corresponding obstruction is zero. Assuming that the obstruction is zero, we choose a lifting to second order (which is not unique) and try to lift this to third order. Again there are obstructions, but if these are zero, the lifting is possible and we can continue. In any case, the obstructions yield formal power series in $K\left[\left[t_{1}, \ldots, f_{n}\right]\right], t_{1}, \ldots, t_{n}$ a $K$-basis of $T_{R}^{1 *}$, and if $J$ denotes the ideal generated by them, $B=K\left[\left[t_{1}, \ldots, t_{m}\right]\right] / J$ will be the base algebra of the formal semi-universal deformation of $R$.

The following proposition describes the module of obstructions to lift a deformation from an Artinian algebra to an infinitesimally bigger one, where we may think of starting with $A=K\left\langle t_{1}, \ldots, t_{n}\right\rangle /\left\langle t_{1}, \ldots, t_{n}\right\rangle^{2}$.

For this, let $R=K\langle x\rangle / I$ and consider a presentation of $I=\left\langle f_{1}, \ldots, f_{k}\right\rangle$,

$$
0 \longleftarrow I \stackrel{\alpha}{\longleftarrow} K\langle x\rangle^{k} \stackrel{\beta}{\longleftarrow} K\langle x\rangle^{\ell}
$$


with $\alpha\left(e_{i}\right)=f_{i}$ and $\operatorname{syz}(I)=\operatorname{ker}(\alpha)=\operatorname{im}(\beta)$ is the module of relations of $f_{1}, \ldots, f_{k}$, which contains the module of Koszul relations,

$$
\operatorname{Kos}=\left\langle f_{i} e_{j}-f_{j} e_{i} \mid 1 \leq i<j \leq j\right\rangle .
$$

Set $\operatorname{Rel}=K\langle x\rangle^{\ell} / \operatorname{ker}(\beta)$ which is isomorphic to $\operatorname{syz}(I)$ and $\operatorname{Rel}_{0}=\beta^{-1}(\operatorname{Kos})$. We define the module $\mathbf{T}_{\mathbf{R}}^{\mathbf{2}}$ by the exact sequence

$$
\operatorname{Hom}_{R}\left(R^{k}, R\right) \stackrel{\beta *}{\longrightarrow} \operatorname{Hom}_{R}\left(\operatorname{Rel} / \operatorname{Rel}_{0}, R\right) \longrightarrow T_{R}^{2} \longrightarrow 0 .
$$

\section{Proposition 3.3.}

1) Let $A^{\prime} \rightarrow A$ be a surjection of Artinian local $K$-algebras with kernel an ideal $J$ satisfying $J^{2}=0$. There is an obstruction map

$$
o b: \operatorname{Def}_{R}(A) \longrightarrow T_{R}^{2} \otimes_{K} J
$$

satisfying: a deformation $A \longrightarrow R_{A}$ of $R$ admits a lifting $A^{\prime} \longrightarrow R_{A^{\prime}}$,

$$
\begin{array}{ccc}
R_{A} & \leftarrow & R_{A^{\prime}} \\
\uparrow & \square & \uparrow \\
A & \leftarrow & A^{\prime},
\end{array}
$$

if and only if $o b\left(\left[A \longrightarrow R_{A}\right]\right)=0\left(\left[A \longrightarrow R_{A}\right]\right.$ denotes the deformation class of $A \longrightarrow R_{A}$ ).

2) If $T_{R}^{1}$ is finite dimensional over $K$ and if $T_{R}^{2}=0$, then the semi-universal deformation $B \longrightarrow R_{B}$ of $R$ has a smooth base space, that is $B$ is a free analytic algebra $K\left\langle t_{1}, \ldots, t_{m}\right\rangle$ for some $m \geq 0$.

For the computation of $T_{R}^{2}$ we choose, as before, a representation

$$
0 \longleftarrow R \longleftarrow K\langle x\rangle \longleftarrow K\langle x\rangle^{k} \stackrel{r}{\longleftarrow} K\langle x\rangle^{\ell} \stackrel{s}{\longleftarrow} K\langle x\rangle^{t} .
$$

Then $\operatorname{Rel}=\operatorname{syz}(I)=\operatorname{Im}(r)$ and $\operatorname{Rel}_{0}$ is the submodule of Rel generated by the $\left(\begin{array}{l}k \\ 2\end{array}\right)$ Koszul relations Kos.

Now Rel $/ \operatorname{Rel}_{0} \longrightarrow R^{k}$ is the induced map defined by the following diagram

$$
\begin{array}{ccccc}
I K\langle x\rangle^{k} & \hookrightarrow & K\langle x\rangle^{k} & \longrightarrow & R^{k} \\
\cup \text { । } & & \cup \text { । } & & \uparrow \\
\operatorname{Rel}_{0} & \hookrightarrow & \operatorname{Rel} & \longrightarrow & \operatorname{Rel} / \operatorname{Rel}_{0} .
\end{array}
$$

To obtain a representation of $\operatorname{Rel} / \operatorname{Rel}_{0}$ we lift the Koszul relations to $K\langle x\rangle^{\ell}$ :

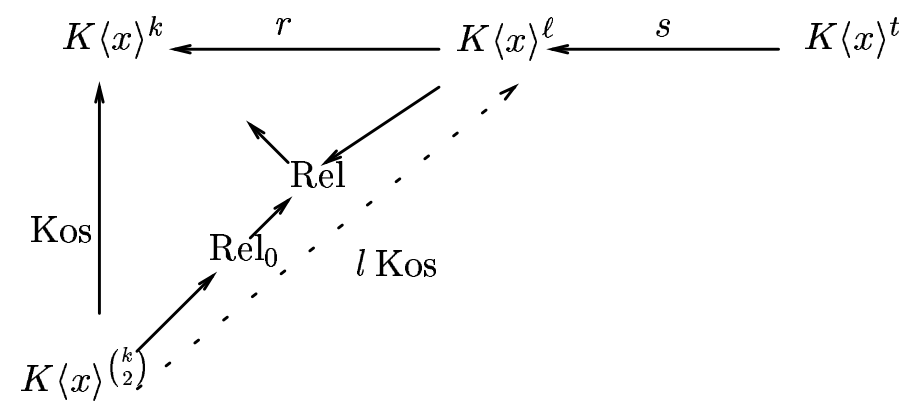


Then $\operatorname{Rel} / \operatorname{Rel}_{0} \simeq K\langle x\rangle^{\ell} / \operatorname{Im}(s)+\operatorname{Im}(\ell \operatorname{Kos})$ and if we denote by $s \ell$ the $\ell \times$ $\left(t+\left(\begin{array}{c}k \\ 2\end{array}\right)\right)$-matrix $s, \ell$ Kos:

$$
0 \longleftarrow \operatorname{Rel} / \operatorname{Rel}_{0} \longleftarrow K\langle x\rangle^{\ell} \stackrel{s \ell}{\longleftarrow} K\langle x\rangle^{t_{1}}, t_{1}=t+\left(\begin{array}{c}
k \\
2
\end{array}\right),
$$

is a representation of $\mathrm{Rel} / \mathrm{Rel}_{0}$.

Now we are interested in a representation of $T_{R}^{2}$ which is just

$$
\operatorname{coker}\left(\operatorname{Hom}_{R}\left(R^{k}, R\right) \longrightarrow \operatorname{Hom}_{R}\left(\operatorname{Rel} / \operatorname{Rel}_{0}, R\right)\right) \text {. }
$$

We dualize the representation of $\operatorname{Rel} / \operatorname{Rel}_{0}$ and obtain

$$
\begin{array}{ccc}
0 \rightarrow \operatorname{Hom}_{K\langle x\rangle}\left(\operatorname{Rel} / \operatorname{Rel}_{0}, R\right) & \rightarrow \operatorname{Hom}_{K\langle x\rangle}\left(K\langle x\rangle^{\ell}, R\right) \stackrel{s \ell^{t}}{\rightarrow} & \operatorname{Hom}_{K\langle x\rangle}\left(K\langle x\rangle^{t_{1}}, R\right) \\
\operatorname{Hom}_{R}\left(\operatorname{Rel} / \operatorname{Rel}_{0}, R\right) & \operatorname{Hom}_{R}\left(R^{\ell}, R\right) & \operatorname{Hom}_{R}\left(R^{t_{1}}, R\right),
\end{array}
$$

that is, $\operatorname{Hom}_{R}\left(\operatorname{Rel} / \operatorname{Rel}_{0}, R\right)=\operatorname{ker}\left(s \ell^{t}\right)$.

Now we take a representation of $\operatorname{Hom}_{R}\left(\operatorname{Rel} / \operatorname{Rel}_{0}, R\right)$

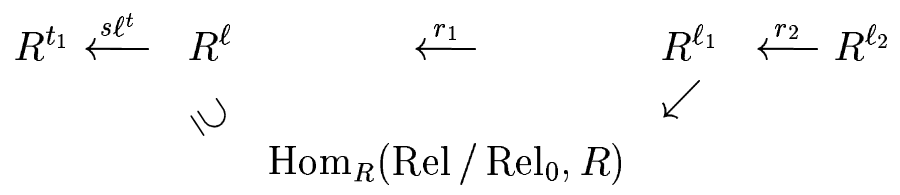

The $\operatorname{map} R^{k} \simeq \operatorname{Hom}_{R}\left(R^{k}, R\right) \longrightarrow \operatorname{Hom}_{R}\left(\operatorname{Rel} / \operatorname{Rel}_{0}, R\right)$ is defined by $r^{t}$ : $R^{k} \longrightarrow R^{\ell}$. We can lift this map to a map ert $: R^{k} \longrightarrow R^{\ell_{1}}$ such that $r_{1} \circ \ell r t=r^{t}$.

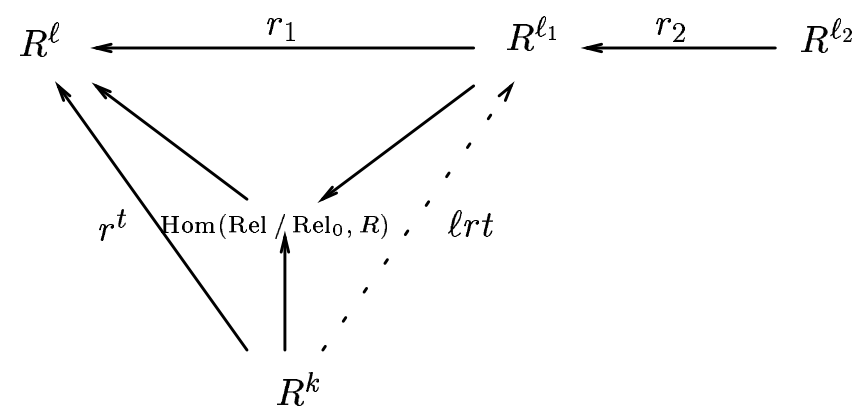

Then $T_{R}^{2}=\operatorname{coker}\left(R^{k} \longrightarrow \operatorname{Hom}\left(\operatorname{Rel} / \operatorname{Rel}_{0}, R\right)\right) \simeq R^{\ell_{1}} / \operatorname{Im}\left(r_{2}\right)+\operatorname{Im}(\ell r t)$ gives the required representation

$$
0 \longleftarrow T_{R}^{2} \longleftarrow R^{\ell_{1}} \stackrel{r_{2} \oplus \ell r t}{\longleftarrow} R^{\ell_{2}} \oplus R^{k} .
$$

We obtain the following algorithm:

\section{Algorithm 3.5.}

$\mathrm{T} 2(I)$

Input: $\quad$ an ideal $I=\left\langle f_{1}, \ldots, f_{k}\right\rangle \subseteq K\langle x\rangle$

Output: a matrix $M \in M_{a, b}(K\langle x\rangle)$ which defines a representation

$$
T_{K\langle x\rangle / I}^{2} \nleftarrow K\langle x\rangle^{a} \stackrel{M}{\longrightarrow} K\langle x\rangle^{b}
$$


- compute $r=\operatorname{syz}(I) \in M_{k, \ell}(K\langle x\rangle)$, the matrix of the syzygies of $f_{1}, \ldots, f_{k}$ and $s=\operatorname{syz}(\operatorname{syz}(I)) \in M_{\ell, t}(K\langle x\rangle)$ to obtain a representation of $K\langle x\rangle / I$;

- compute the matrix $\operatorname{Kos} \in M_{k,\left(\begin{array}{c}k \\ 2\end{array}\right)}$, the Koszul matrix of the relations of $f_{1}, \ldots, f_{k}$

- lift Kos to a matrix $\ell$ Kos $\in M_{\ell,\left(\begin{array}{l}k \\ 2\end{array}\right)}$ such that $r \cdot \ell$ Kos $=$ Kos;

- concatenate $\ell$ Kos and $s$ to obtain the matrix $s \ell=\ell \operatorname{Kos}, s \in M_{\ell,\left(\begin{array}{l}k \\ 2\end{array}\right)+t}$;

- compute in $K\langle x\rangle / I$ a representation of the kernel $\operatorname{ker}\left(s \ell^{t}\right)$ given by matrices $r_{1}$ and $r_{2}\left(r_{1}=\operatorname{syz}\left(s \ell^{t}\right) \in M_{\ell, \ell_{1}}(K\langle x\rangle / I), r_{2}=\operatorname{syz}\left(r_{1}\right) \in\right.$ $\left.M_{\ell_{1}, \ell_{2}}(K\langle x\rangle / I)\right)$;

- lift the matrix $r^{t}$ to a matrix $\ell r t \in M_{\ell_{1}, k}(K\langle x\rangle / I)$ such that $r_{1} \cdot \ell r t=r^{t}$;

- concatenate $\ell r t$ and $r_{2}$ to obtain $t 2=\ell r t, r_{2} \in M_{\ell_{1}, k+\ell_{2}}(K\langle x\rangle / I)$;

- choose a matrix $M_{0} \in M_{\ell_{1}, k+\ell_{2}}(K\langle x\rangle)$ such that $M_{0} \bmod I=t 2$;

- choose a matrix $L \in M_{\ell_{1}, k \cdot \ell_{1}}$ (corresponding to $I K\langle x\rangle^{\ell_{1}}$ ) such that

$$
0 \leftarrow(K\langle x\rangle / I)^{\ell_{1}} \leftarrow K\langle x\rangle^{\ell_{1}} \stackrel{L}{\longleftarrow} K\langle x\rangle^{k \cdot \ell_{1}}
$$

is exact;

- concatenate $M_{0}$ and $L$ to obtain $M=M_{0}, L$;

- $\operatorname{return} M$.

\section{References}

[Ar] Artin, M.: Lectures on deformations of singularities. Tata Institute, Bombay (1976).

[Bu1] Buchberger, B.: Ein Algorithmus zum Auffinden der Basiselemente des Restklassenringes nach einem nulldimensionalen Polynomideal. PhD Thesis, University of Innsbruck, Austria (1965).

[Bu2] Buchberger, B.: Ein algorithmisches Kriterium für die Lösbarkeit eines algebraischen Gleichungssystems. In: Aequ. Math. 4, 374-383 (1970).

[Bu3] Buchberger, B.: Gröbner bases: an algorithmic method in polynomial ideal theory. In: Recent trends in multidimensional system theory, N.B. Bose, ed., Reidel (1985). 
[Bu4] Buchberger, B.: Introduction to Gröbner bases. This volume.

[BWe] Becker, T.; Weispfennig, V.: Gröbner Bases, A Computational Approach to commutative Algebra. Graduate Texts in Mathematics 141, Springer 1993.

[CLO] Cox, D.; Little, J.; O'Shea, D.: Ideals, Varieties and Algorithms. Springer Verlag (1992).

[CNR] Capani, A.; Niesi, G.; Robbiano, L.: Some Features of CoCoA 3. Moldova Journal of Computer Science. To appear.

[Ei] Eisenbud, D: Commutative Algebra with a view toward Algebraic Geometry. Springer 1995.

[ERS] Eisenbud, D.; Riemenschneider, O.; Schreyer, F.-O.: Resolutions of Cohen-Macaulay Algebras. Math Ann.257 (1981).

[Getal] Grassmann, H.; Greuel, G.-M.; Martin, B.; Neumann, W.; Pfister, G.; Pohl, W.; Schönemann, H.; Siebert, T.: Standard bases, syzygies and their implementation in SINGULAR. In: Beiträge zur angewandten Analysis und Informatik, Shaker, Aachen, 6996, 1994.

[GP] Greuel, G.-M.; Pfister,G.: Advances and improvements in the theory of standard bases and syzygies. Arch. Math. 66, 163-1796 (1996).

[GPS] Greuel, G.-M.; Pfister, G.; Schönemann, H.: SINGULAR Reference Manual, Reports On Computer Algebra Number 12, May 1997, Centre for Computer Algebra, University of Kaiserslautern from www.mathematik.uni-kl.de/zca/Singular.

[GR] Grauert, H.; Remmert, R.: Analytische Stellenalgebren. Springer 1971.

[Gr] Grauert, H.: Über die Deformation isolierter Singularitäten analytischer Mengen. Invent. Math. 15, 171-198 (1972).

[Gra] Gräbe, H.-G.: The tangent cone algorithm and homogeneization. J. Pure Appl. Alg. 97, 303-312 (1994).

[GS] Grayson, D.; Stillmann, M.: A computer software system designed to support research in commutative algebra and algebraic geometry. Available from math.uiuc.edu.

[GT] Gianni, P.; Trager, B.: Integral closure of noetherian rings. Preprint, to appear. 
[Hi] Hironaka, H.: Resolution of singularities of an algebraic variety over a field of characteristic zero. Ann. of Math. 79, 109-326 (1994).

[J] de Jong, T.: An algorithm for computing the integral closure. Preprint, Saarland University, Saarbrücken.

[Ku] Kunz, E.: Einführung in die kommutative Algebra und algebraische Geometrie. Vieweg (1980).

[La] Laudal, O.A.: Formal Moduli of Algebraic Structures. LNM 754, Springer (1979).

[Mil] Milnor, J.: Singular Points of Complex Hypersurfaces. Ann. of Math. Studies 61, Princeton (1968).

[Mo] Mora, T.: An algorithm to compute the equations of tangent cones. Proc. EUROCAM 82, Lecture Notes in Comput. Sci. (1982).

[MM] Möller, H.M.; Mora, T.: Computational aspects of reduction strategies to construct resolutions of monomial ideals. Proc. AAECC 2, Lecture Notes in Comput. Sci. 228 (1986).

[MPT] Mora, T.; Pfister, G.; Traverso, C.: An introduction to the tangent cone algorithm. In: Issues in non-linear geometry and robotics, JAI Press (1992).

[Sch1] Schreyer, F.-O.: Die Berechnung von Syzygien mit dem verallgemeinerten Weierstrass'schen Divisionssatz. Diplomarbeit, Hamburg (1980).

[Sch2] Schreyer, F.-O.: A standard basis approach to syzygies of canonical curves. J. reine angew. Math. 421, 83-123 (1991).

[Sch3] Schreyer, F.-O.: Syzygies of canonical curves and special linear series. Math. Ann. 275 (1986).

[Schl1] Schlessinger, M.: Functors of Artin rings. Trans. AMS 130, 208$222,(1968)$.

[Schl2] Schlessinger, M.: On rigid singularities. Rice. Univ. Stud. 59, 147162 (1973).

[St] Sturmfels, B.: Algorithms in Invariant Theory. Springer Verlag (1993).

[V] Vasconcelos, W.: Computing the integral closure of an affine domain. Proc. AMS 113 (3), 633-638 (1991). 